\title{
BOUNDARY BEHAVIOR OF CAPILLARY SURFACES POSSIBLY WITH EXTREMAL BOUNDARY ANGLES
}

FEI-TSEN LIANG

Received 7 April 2005 and in revised form 25 October 2005

For solutions to the capillarity problem possibly with the boundary contact angle $\theta$ being 0 and/or $\pi$ in a relatively open portion of the boundary which is $C^{2}$, we will show that if the solution is locally bounded up to this portion of boundary, the trace of the solution on this portion is piecewise Lipschitz continuous and the solution is Hölder continuous up to the boundary, provided the prescribed mean curvature is bounded from above and from below. In the case where $\theta$ is not required to be bounded away from $\pi / 2,0$, and $\pi$, and the mean curvature $H\left(x, t_{0}\right)$ belongs to $L^{p}(\Omega)$ for some $t_{0} \in \mathbb{R}$ and $p>n$, under the assumption that in a neighborhood of a relatively open portion of the boundary the solution is of rotational symmetry, the trace of the solution on this portion of the boundary is shown to be Hölder continuous with exponent $1 / n$ if $n \geq 3$ and with exponent $1 / 3$ if $n=2$.

\section{Introduction}

Given a domain $\Omega \subset \mathbb{R}^{n}$, we are interested in regularity near the boundary $\partial \Omega$ for solutions $u \in C^{2}(\Omega)$ of the mean curvature equation

$$
\operatorname{div} T u=H(x, u) \quad \text { on } \Omega,
$$

subject to the "contact angle" boundary condition

$$
T u \cdot v=\cos \theta \quad \text { on } \partial \Omega
$$

for a piecewise continuous function $\theta$ which takes its values in $[0, \pi]$, where

$$
T u=\frac{D u}{\sqrt{1+|D u|^{2}}}
$$

and $\nu$ is the outward-pointing unit normal of $\partial \Omega$. Thus, geometrically, we are looking for a function $u$ on $\bar{\Omega}$ whose graph has the prescribed mean curvature $H$ and which meets the cylinder over the boundary in the prescribed angle $\theta$. The function $H$ is assumed to 
be locally Lipschitz continuous on $\bar{\Omega} \times \mathbb{R}$ satisfying the structural conditions

$$
\frac{\partial H}{\partial t}(x, t) \geq 0, \quad \text { for } x \in \Omega, t \in \mathbb{R}
$$

One of our main interests is in the case when $\theta$ takes the value 0 and/or $\pi$ in a relatively open portion of $\partial \Omega$, at which the solution graph is required to meet the boundary cylinder vertically upward or downward; thus, when approaching this portion, the Euclidean norm of the gradient becomes unbounded, which makes this case mathematically more subtle than the case when $\theta$ is bounded away from 0 and $\pi$. We will show in this paper that if this portion of $\partial \Omega$ is $C^{2}$ and if the prescribed mean curvature is bounded from above and from below, then if the solution is bounded locally up to this portion of the boundary, the trace of the solution on this portion is piecewise Lipschitz continuous and the solution is Hölder continuous up to the boundary.

For the regularity result, no a priori regularity is imposed at the boundary and write the equation together with the contact angle boundary condition in the weak form as follows: let $\nu$ be any $C^{0}(\Omega)$ function which coincides on $\partial \Omega$ with the outward-pointing unit normal of $\partial \Omega$ and let $\theta: \partial \Omega \rightarrow[0, \pi]$ be a given function which is measurable with respect to $(n-1)$-dimensional Hausdorff measure $\mathcal{H}_{n-1}$ on $\partial \Omega \cap B_{\rho}$. Suppose that

$$
u \in C^{2}(\Omega) \cap W^{1,1}(\Omega)
$$

We say $u$ satisfies the contact angle problem

$$
\begin{gathered}
\operatorname{div} T u=H(x, u) \quad \text { in } \Omega, \\
T u \cdot v=\cos \theta \quad \text { on } \partial \Omega
\end{gathered}
$$

in the weak sense provided that

$$
\int_{\Omega}(T u \cdot D \eta+H(x, u) \eta) d x=\int_{\partial \Omega} \cos \theta \cdot \eta d \mathcal{H}_{n-1}, \quad \text { for each } \eta \in W^{1,1}(\Omega) .
$$

We will establish the following theorem.

MaIn Theorem 1.1. Suppose $\theta \equiv 0$ or $\theta \equiv \pi$ in a relatively open subset $\tilde{\partial} \Omega$ of $\partial \Omega$ and $\Omega \subset$ $\mathbb{R}^{n}$. If $\tilde{\partial} \Omega$ is $C^{2}$ and if the prescribed mean curvature $H(x, t)$ is locally Lipschitz continuous on $\Omega \times \mathbb{R}$, satisfies (1.4), and is bounded in absolute value by a constant $H_{*}$,

$$
|H(x, u)| \leq H_{*}, \quad \text { for } x \in \Omega \text {. }
$$

Let $u \in C^{2}(\Omega) \cap W^{1,1}(\Omega)$ be a solution of (1.1) and (1.2) in the weak sense. Then the trace of $u$ on $\tilde{\partial} \Omega$ is Lipschitz continuous with Lipschitz norm

$$
\left(2^{n+6}(n+1)+2\right)\left(\frac{3 \omega_{n}}{n}\right)\left(1+2 H_{*}\left(\sup _{\Omega \cap A_{\delta_{0}}\left(x_{0}\right)} u-\inf _{\Omega \cap A_{\delta_{0}}\left(x_{0}\right)} u\right) \cdot H_{*}\right)
$$


which is completely determined by $H_{*}, n$, and $\left(\sup _{\Omega} u-\inf _{\Omega} u\right)$. Furthermore, $u$ is Hölder continuous up to $\tilde{\partial} \Omega$ with exponent determined by the same set of quantities.

We notice that the last statement follows from [14].

Suppose that $\theta, \partial \Omega$ are of the rotational symmetry with respect to the same symmetry axis. Furthermore, suppose that the same rotational symmetry of the function $H(x, z)$ is imposed on the first variable for every value of $t$.

We will establish in Section 4 the following, which is valid in particular in the case where $\theta$ is not required to be bounded away from $\pi / 2,0$, and $\pi$.

Main Theorem 1.2. Suppose that $\tilde{\partial} \Omega$ is a relatively open subset of $\partial \Omega$ which is $C^{2}$. Let $\beta=\cos \theta$. Suppose that, at $x_{0} \in \tilde{\partial} \Omega$, we have $\left(\beta x_{0}\right)=0$, and is Lipschitz continuous in $\tilde{\partial} \Omega$ with Lipschitz constant $L$. Suppose that in a neighborhood $\tilde{U}$ of $\tilde{\partial} \Omega$ in $\Omega$, the function $\left.u\right|_{\tilde{U}}$ is of rotational symmetry, and suppose that the prescribed mean curvature $H(x, t)$ is locally Lipschitz continuous on $\Omega \times \mathbb{R}$, satisfies (1.4), and

$$
H\left(x, t_{0}\right) \in L^{p}(\Omega), \quad \text { for some } p, p>n, t_{0} \leq u\left(x_{0}\right) .
$$

Let $u \in C^{2}(\Omega) \cap W^{1,1}(\Omega)$ be a solution of (1.1) and (1.2) in the weak sense. Then the trace of $u$ on $\tilde{\partial} \Omega$ is Hölder continuous with exponent $1 / n$. The Hölder norm is determined by $n$ and $\left(\sup _{\Omega} u-\inf _{\Omega} u\right), L$, and $H\left(x_{0}\right)$.

\section{Preliminaries}

2.1. The following result is obtained by Korevaar and Simon in [8].

Proposition 2.1. Suppose that $H(x, t)$ is locally Lipschitz in $\Omega \times \mathbb{R}$, satisfies (1.4), and

$$
\left|\frac{\partial H}{\partial t}\right|+\sum_{i=1}^{n}\left|\frac{\partial H}{\partial x_{i}}\right| \leq \Lambda, \quad \text { for } x \in \Omega, z \in \mathbb{R}
$$

Suppose that $u \in C^{2}\left(\Omega \cap B_{\rho}\right) \cap W^{1,1}\left(\Omega \cap B_{\rho}\right)$ satisfies

$$
\int_{\Omega \cap B_{\rho}}(T u \cdot D \eta+H(x, u) \eta) d x=\int_{\partial \Omega \cap B_{\rho}} \cos \theta \cdot \eta d \mathscr{H}_{n-1}, \quad \text { for each } \eta \in W^{1,1}\left(\Omega \cap B_{\rho}\right),
$$

with $\theta \equiv 0$ on $\partial \Omega \cap B_{\rho}$. Suppose that $\partial \Omega \cap B_{\rho}$ is of class $C^{2}$ and $u$ is bounded locally up to the boundary in $\bar{\Omega} \cap B_{\rho}$. Then,

$$
T^{*} u \equiv \frac{(D u,-1)}{\sqrt{1+|D u|^{2}}} \in C^{0}\left(\bar{\Omega} \cap B_{\rho / 2}\right)
$$


and $u$ satisfies the boundary condition in a classical way (i.e., (1.2) holds with $\theta \equiv 0$ on $\left.\partial \Omega \cap B_{\rho / 2}\right)$. Furthermore, considered as a function on the graph of $\left.u\right|_{\Omega \cap B_{\rho / 2}}, T^{*} u$ is Lipschitz continuous. Furthermore,

$$
\text { (closure graph } u) \cap\left(\Omega \cap B_{\rho / 2}\right) \times \mathbb{R}
$$

is contained in a properly embedded $C^{1,1}$ submanifold of $\mathbb{R}^{n+1}$.

We notice that this result does not yield the regularity of the trace of the solution obtained in this present work.

2.2. Simon and Spruck treat in [15] the case where $\Omega$ is $C^{4}, \theta$ in (1.2) is $C^{1, \alpha}$ for some $0<\alpha<1$, and $H(x, t)$ is strictly monotone in $t$ :

$$
\inf _{x \in \bar{\Omega} ; t \in \mathbb{R}} \frac{\partial H}{\partial t}(x, t)>0
$$

In case $0<\theta<\pi$, the existence of a solution $u \in C^{2}(\bar{\Omega})$ of (1.1) and (1.2) is established in [15]. In case $\theta$ is allowed to take the values 0 and/or $\pi$, setting

$$
\begin{gathered}
S_{1}^{+}=\{x: x \in \partial \Omega, \theta \equiv 0 \text { in some neighborhood of } x\} \\
S_{1}^{-}=\{x: x \in \partial \Omega, \theta \equiv \pi \text { in some neighborhood of } x\} \\
S_{2}=\{x: x \in \partial \Omega, 0<\theta<\pi\}
\end{gathered}
$$

a function $u \in C^{2}\left(\Omega \cup S_{2}\right)$ is shown to exist in [15] which satisfies (1.1) in $\Omega$ and satisfies (1.2) on $S_{2}$; furthermore, $u$ is Hölder continuous at each point of $S_{1}^{+} \cup S_{1}^{-}$, has a restriction to $\partial \Omega$ which is Lipschitz continuous at each point of $S_{1}^{+} \cup S_{1}^{-}$, and (1.2) is satisfied on $S_{1}^{ \pm}$ in the sense that

$$
\lim _{\mathcal{\varepsilon}^{+} \rightarrow 0} \int_{U \cap \Omega_{\varepsilon}}|T u \cdot \nu \pm 1| d x=0, \quad \text { for each } U \subset \Omega \text { with } \bar{U} \cap \partial \Omega \subset S_{1}^{\mp},
$$

assuming that $T u$ is extended to some boundary strip $\Omega_{\varepsilon}$ with width $\varepsilon$ so that it is constant along the normals to $\partial \Omega$. This regularity result is obtained by first establishing estimates of the tangential derivatives under the condition that $|\cos \theta| \leq \gamma<1$ for some positive constant $\gamma$; in case $\theta$ is constant in a neighborhood of the point under consideration, this estimate of tangential derivatives is independent of $\gamma$. This proves the Lipschitz continuity of the trace of $u$ on $\partial \Omega$, which yields Hölder continuity of $u$ together with [14, Theorem 2]. Estimates for the tangential derivatives are obtained by performing a transformation of coordinates near the boundary analogously to that in [14], together with a subsequent differentiation of (1.1), (1.2), and inserting (2.5) into the resultant identities. The disadvantage of these proofs is that $H$ is supposed to satisfy the strict inequality (2.5) rather than the less restrictive condition (1.4). 
2.3. In contrast, the following estimates for the boundary oscillation of $u$ is established in [10, Main Theorem III].

Proposition 2.2. Let $u \in C^{2}(\Omega) \cap W^{1,1}(\Omega)$ be a bounded solution to (1.1) and (1.2) in the weak sense of (1.4). Suppose that for positive constants $\tilde{\beta}, \tilde{\tilde{\beta}} \leq 1$ and a ball $B_{R}\left(x_{0}\right)$ intersecting the interior of $\Omega$, the function $\cos \theta$ is continuous on $\partial \Omega \cap B_{R}\left(x_{0}\right)$ and such that either of the inequalities

$$
\tilde{\tilde{\beta}} \leq \cos \theta \leq \tilde{\beta}, \quad-\tilde{\tilde{\beta}} \geq \cos \theta \geq-\tilde{\beta}
$$

holds for all $x \in \partial \Omega \cap B_{R}\left(x_{0}\right)$, and such that

$$
\begin{gathered}
\hat{H}^{ \pm}(x)=H\left(x, \pm \inf _{\partial \Omega} u\right) \in L^{p}(\Omega), \quad \hat{\hat{H}}^{ \pm}(x)=H\left(x, \pm \sup _{\partial \Omega} u\right) \in L^{p}(\Omega), \\
H(x, 0) \in L^{1}(\Omega) .
\end{gathered}
$$

Suppose $\partial \Omega$ is piecewise Lipschitz continuous with possible outward and/or inward cusps. Then the trace of $u$ on $\partial \Omega$ is Lipschitz continuous in $\partial \Omega \cap B_{R}\left(x_{0}\right)$ if $\partial \Omega \cap B_{R}\left(x_{0}\right)$ is either $C^{2}$ or is the graph of a Lipschitz continuous function with Lipschitz constant L such that $\tilde{\beta} \sqrt{1+L^{2}}<1$. The Lipschitz constant of the trace of $u$ on $\partial \Omega \cap B_{R}\left(x_{0}\right)$ depends only on $H$, $n$, together with the constants $\tilde{\beta}, \tilde{\tilde{\beta}}$, and $\mathcal{K}_{\partial \Omega \cap \overline{B_{R}\left(x_{0}\right)}}$, where $\mathcal{K}_{\partial \Omega \cap \overline{B_{R}\left(x_{0}\right)}}$ is an upper bound for the absolute value of the principal curvatures of $\partial \Omega \cap B_{R}\left(x_{0}\right)$ in case $\partial \Omega \cap B_{R}\left(x_{0}\right)$ is $C^{2}$ and $\mathcal{Y}_{\partial \Omega \cap \overline{B_{R}\left(x_{0}\right)}}=\sqrt{1+L^{2}}$ in case $\partial \Omega \cap B_{R}\left(x_{0}\right)$ is Lipschitz continuous with Lipschitz constant L.

We notice that (2.9) hold in particular if $|H(x, t)|$ is bounded in $\bar{\Omega} \times \mathbb{R}$.

This result is established by modifying the approach taken in $[3,4,5]$, which is based on the minimizing property satisfied by $u$ and the following result due to Stampacchia [16, Lemma 4.1].

Lemma 2.3 (Stampacchia). Suppose that $\phi(t)$ is a nonnegative nondecreasing function defined on $\mathbb{R}$ such that for some constant $C, k_{0}$, and $\gamma$, there holds

$$
(h-k) \cdot \phi(t) \leq C \cdot[\phi(k)]^{\gamma}, \text { for each } h>k \geq k_{0} .
$$

Then,

$$
\begin{gathered}
h^{1 /(1-\gamma)} \cdot \phi(h) \leq 2^{1 /(1-\gamma)^{2}} \cdot\left\{C^{1 /(1-\gamma)}+\left(2 k_{0}\right)^{1 /(1-\gamma)} \cdot \phi(k)\right\}, \quad \text { if } \gamma<1, \\
\phi\left(k_{0}+\tau\right)=0, \quad \text { if } \gamma>1,
\end{gathered}
$$

where

$$
\tau=2^{\gamma /(\gamma-1)} \cdot C \cdot\left[\phi\left(k_{0}\right)\right]^{\gamma-1} .
$$

Let $\phi(t)$ be the area of the level set of a function $\eta u$, where $\eta$ is a suitably chosen cutoff function in a suitably chosen domain. The identity (2.12) is applied to prove [10, Proposition 2], once the inequality (2.11) is shown to hold for some $\gamma>1$. To make this feasible, the condition (2.8) is crucial. At neighborhoods of points where $\theta=0, \pi$, or $\pi / 2$, 
the condition (2.8), however, is not satisfied. Main Theorem 1.1 treats the cases that $\theta \equiv 0$ or $\pi$ in a relatively open subset of $\partial \Omega$. Main Theorem 1.2 treats the case that $\theta=\pi / 2$ at a point of $\partial \Omega$ near which $\beta$ is Lipschitz continuous.

The proof of Main Theorem 1.2 will be based on another modification of the technique used in $[3,4,5]$. The inequalities $(2.10)$ will be shown to hold for some $\gamma_{i}<1$, $i=0,1, \ldots$, iteratively with $\phi$ being the level set of some function $\eta_{i} u$, where $\eta_{i}$ are suitably chosen cut-off functions in some suitably chosen domains depending on $i$. Using (2.11), the exponent $\gamma_{i}$ will strictly increase as the number of times $i$ of iteration increases. After a finite number of times of iteration, the exponent becomes greater than 1 and the identity (2.12) can be applied.

We will obtain the inequality (4.32) below. In order to estimate the third and last terms on the right-hand side of (4.32), we have to control the size of the level set of the function $\eta_{i} u$. For this purpose, we impose the restrictions that $\Omega, \beta$, and hence $u$ are of the same rotational symmetry. The rotational symmetry of $\Omega$ enables us to choose $\eta_{i}$ to be of the same rotational symmetry, and hence control the size of the level set of the function $\eta_{i} u$. In order to estimate the fifth and sixth terms on the right-hand side of (4.32), we impose the restriction that $\beta\left(x_{0}\right)=0$ and $\beta$ is Lipschitz continuous in a neighborhood of $x_{0}$ or $\partial \Omega$.

2.4. As for the regularity near the boundary, the following result is established in [11] in which the region $A_{r}(\hat{x})$, for small positive number $r$, is chosen as follows. Namely, setting

$$
\partial^{*} \Omega_{t}=\{x: x \in \Omega, \operatorname{dist}(x, \partial \Omega)=t\} \quad \text { for } t>0,
$$

we let the boundary $\partial\left(\Omega \cap A_{r}(\hat{x})\right)$ be made up of three parts:

$$
\left(\partial \Omega \cap \overline{A_{r}(\hat{x})}\right) \cup\left(\partial^{*} A_{r}(\hat{x})\right) \cup\left(\partial^{* *} A_{r}(\hat{x})\right),
$$

such that

$$
\begin{gathered}
\partial^{*} A_{r}(\hat{x}) \subset \partial^{*} \Omega_{r}, \quad \partial^{* *} A_{r}(\hat{x})=\left(\partial A_{r}(\hat{x}) \cap \Omega\right) \backslash \partial^{*} \Omega_{r}, \\
\left.D d \cdot \nu_{\Omega \cap A_{r}(\hat{x})}\right|_{\partial^{* *} A_{r}(\hat{x})}=0,
\end{gathered}
$$

where we let $\nu_{\Omega \cap A_{r}(\hat{x})}$ be the unit outward normal to $\partial\left(\Omega \cap A_{r}(\hat{x})\right)$; furthermore,

$$
\operatorname{diam}\left(\partial \Omega \cap \overline{A_{r}(\hat{x})}\right) \leq r, \quad \operatorname{diam}\left(\partial^{*} A_{r}(\hat{x})\right) \leq r
$$

and $\partial \Omega \cap A_{r}(\hat{x})$ and $\partial^{*} A_{r}(\hat{x})$ include balls of radius $(r / 2)^{n-1}$ centered, respectively, at $\hat{x}$ and a point at distance $r$ from $\hat{x}$. We established in [11] the following proposition. 
Proposition 2.4. Let $u \in C^{2}(\Omega) \cap W^{1,1}(\Omega)$ be a solution to (1.1) and (1.2) in the weak sense. Let $|H(x, t)|$ be bounded by the constant $H_{*}$ in $\bar{\Omega} \times \mathbb{R}$. Suppose that for a point $x_{0} \in$ $\partial \Omega$, there exist positive constants $\tilde{\beta}, \tilde{\tilde{\beta}}<1$, and a sufficiently small positive number $\delta_{0}$ such that one of the inequalities in (2.8) holds for $x \in \partial \Omega \cap \overline{A_{\delta_{0}}\left(x_{0}\right)}$. Suppose that $\delta_{0}$ is so small that $\partial \Omega \cap A_{\delta_{0}}\left(x_{0}\right)$ is of class $C^{2}$. Then $u$ is Lipschitz continuous in $\Omega \cap A_{\delta_{0}}\left(x_{0}\right)$ up to the boundary; for each positive number $\tau$, the Lipschitz norm of $u$ in $\overline{\Omega \cap A_{\delta_{0}}\left(x_{0}\right)}$ is determined by $\tau, H_{*}, n, \tilde{\beta}, \tilde{\beta}, \mathscr{K}_{\partial \Omega \cap \overline{A_{\delta_{0}}\left(x_{0}\right)}}$, and $|\Omega|$; here $\mathscr{K}_{\partial \Omega \cap \overline{A_{\delta_{0}}\left(x_{0}\right)}}$ is an upper bound for the absolute value of the principal curvatures of $\partial \Omega \cap A_{\delta_{0}}\left(x_{0}\right)$.

2.5. The main part of [11] is devoted to estimating the $L^{1}$-norm of $u$ which begins with writing (1.1) and (1.2) in weak form (1.7) in which the assumed boundedness of $|u|$ allows us to take the test function to be $\left(u(x)-\inf _{\Omega} u\right)$ or $\left(\sup _{\Omega} u-u(x)\right)$. The resultant inequalities suggest to us to restrict $u$ to a small region $\Omega \cap A$ of the type indicated in the beginning of [11, Section 3], which is analogous to that of $A_{r}\left(x_{0}\right)$ above. In [11, Section 4], a modified version of Sobolev inequality is applied to obtain estimates of $\int_{\Omega \cap A_{\delta_{0}}\left(x_{0}\right)}\left(\sup _{\Omega \cap A_{\delta_{0}}\left(x_{0}\right)} u-u(x)\right) d x$ and $\int_{\Omega \cap A_{\delta_{0}}\left(x_{0}\right)}\left(u(x)-\inf _{\Omega \cap A_{\delta_{0}}\left(x_{0}\right)} u\right) d x$. In order to apply this modified version of Sobolev inequality, we have to apply the condition (2.8) to

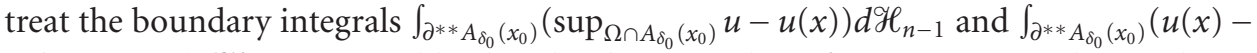
$\left.\inf _{\Omega \cap A_{\delta_{0}}\left(x_{0}\right)} u\right) d \mathcal{H}_{n-1}$. At neighborhoods of points where $\theta=0, \pi$, or $\pi / 2$, the condition (2.8), however, is not satisfied.

The proof of Proposition 2.4 is based on a modification of the reasoning by Giusti [7, pages 312-313], which leads to estimates for the oscillation of $u$ in terms of the $L^{1}$ norm of $u$, under the conditions that either the subgraph of $u$ or the complement of the subgraph of $u$ includes a large portion of a sufficiently small cylinder-type region around $u\left(x_{0}\right), x_{0} \in \partial \Omega$. Such conditions are proved in [7, Theorem 3.2] to be fulfilled by capillary surfaces with boundary contact angle bounded away from 0 or $\pi$. In order to prove Main Theorem 1.1, we will rephrase this result in [7] as Theorem 3.1 below.

\section{Proof of Main Theorem 1.1}

3.1. Estimates of the oscillation of $u$ in terms of the $L^{1}$-norm of $u$.

3.1.1. Equation (1.1) is the Euler equation of the functional

$$
\mathscr{F}_{*}(v)=\int_{\Omega} \sqrt{1+|D v|^{2}} d x+n \int_{\Omega} \int_{0}^{v} H(x, t) d t d x,
$$

and corresponding to the capillarity problem with boundary contact angle $\theta$ is the problem of minimizing the functional

$$
\mathscr{F}_{*}=\mathscr{F}_{*}(v)+\int_{\partial \Omega}(\cos \theta) v d \mathscr{H}_{n-1}
$$

among all $v \in \mathrm{BV}(\Omega)$.

Miranda [13] introduced the notion of generalized solutions for the minimal surface equation. Giusti $[6,7]$ used the analogous notion of generalized solutions for the mean curvature equation. The idea of generalized solutions originates from the observation that 
3932 Boundary behavior of capillary surfaces

a function $u: \Omega \rightarrow \mathbb{R}$ is a solution of (1.1) if and only if its subgraph

$$
U=\{(x, t) \in \Omega \times \mathbb{R}, t<u(x)\}
$$

minimizes the functional

$$
\mathbb{F}_{*}(U)=\int_{\Omega \times \mathbb{R}}\left|D \phi_{U}\right|+\int_{\Omega \times \mathbb{R}} H \phi_{U} d x d t
$$

locally in $\Omega \times \mathbb{R}$, in the sense that for every $V$ coinciding with $U$ outside some compact set $K \subset \Omega \times \mathbb{R}$, we have

$$
\int_{K}\left|D \phi_{U}\right|+\int_{K} H \phi_{U} d x d t \leq \int_{K}\left|D \phi_{V}\right|+\int_{K} H \phi_{V} d x d t
$$

here and in the following, $\phi_{V}$ is the characteristic function of the set $V$ :

$$
\phi_{V}(x, t)= \begin{cases}1 & \text { if }(x, t) \in V \\ 0 & \text { if }(x, t) \notin V .\end{cases}
$$

Moreover, a function $u \in \operatorname{BV}(\Omega)$ minimizes $\mathscr{F}$ in $\Omega$ if and only if its subgraph minimizes the functional

$$
\mathbb{F}(U)=\int_{\Omega \times \mathbb{R}}\left|D \phi_{U}\right|+\int_{\Omega \times \mathbb{R}} H \phi_{U} d x d t+\int_{\partial \Omega \times \mathbb{R}}(\cos \theta) \phi_{U} d \mathscr{H}_{n} .
$$

Minimization is here to be understood in the following sense: for $T>0$, set

$$
\begin{gathered}
Q=\Omega \times[-T, T], \\
\delta Q=\partial \Omega \times[-T, T],
\end{gathered}
$$

and for $U \subset Q$,

$$
\mathbb{F}_{T}(U)=\int_{Q_{T}}\left|D \phi_{U}\right|+\int_{Q_{T}} H \phi_{U} d x d t+\int_{\delta Q_{T}}(\cos \theta) \phi_{U} d \mathcal{H}_{n} .
$$

We say that $U$ minimizes $\mathbb{F}_{T}$ in $Q_{T}$ if

$$
\mathbb{F}_{T}(U) \leq \mathbb{F}_{T}(S)
$$

for every Caccioppoli set $S \subset Q_{T}$. We say that $U$ minimizes $\mathbb{F}$ in $\Omega \times \mathbb{R}$ if $U$ minimizes $\mathbb{F}_{T}$ in $Q_{T}$ for every $T>0$. A function $u: \Omega \rightarrow[-\infty, \infty]$ is called a generalized solution of (1.1) in $\Omega$ if its subgraph $U$ minimizes the functional $\mathbb{F}_{*}$ locally in $\Omega \times \mathbb{R}$. A function $u: \Omega \rightarrow[-\infty, \infty]$ is called a generalized solution of the functional $\mathscr{F}$ if its subgraph $U$ minimizes the functional $\mathbb{F}$ in $\Omega \times \mathbb{R}$. 
A generalized solution can take the values $\pm \infty$ on sets of positive measure. However, a locally bounded generalized solution $u(x)$ is a classical solution of (1.1) in $\Omega$. A bounded generalized solution of the functional $\mathscr{F}$ is a solution of (1.1) and (1.2) in the weak sense. Thus, by the uniqueness (up to an additive constant) of the weak solution of (1.1) and (1.2) in the sense (1.7) (cf. [2, Theorems 7.6 and 7.9]), each weak solution of (1.1) and (1.2) in the sense (1.7) is a generalized solution of the functional $\mathscr{F}_{*}$.

Let $u$ be a weak solution to (1.1) and (1.2) in the sense (1.4). For points $\hat{z}=(\hat{x}, \hat{t}) \in$ $\Omega \times \mathbb{R}$, we now consider the sets

$$
\begin{aligned}
& \widetilde{C}_{\varepsilon}^{+}(\hat{z})=\{(x, t):|u(x)-u(\hat{x})|<\varepsilon, 0<t-u(\hat{x})<\varepsilon\}, \\
& \widetilde{C}_{\varepsilon}^{-}(\hat{z})=\{(x, t):|u(x)-u(\hat{x})|<\varepsilon, 0<u(\widehat{x})-t<\varepsilon\},
\end{aligned}
$$

and let

$$
\tilde{U}_{\varepsilon}^{+}(\hat{z})=U \cap \widetilde{C}_{\varepsilon}^{+}(\hat{z}), \quad \widetilde{U}_{\varepsilon}^{-}(\hat{z})=U \cap \widetilde{C}_{\varepsilon}^{-}(\hat{z}) .
$$

Based on the minimizing property of the subgraph of the function $u$, the following result can be established, whose proof is essentially identical with that of corresponding results in [7].

Theorem 3.1. Let $u$ be a weak solution to (1.1) and (1.2) in the sense (1.7). Let $U$ be the subgraph of $u$. Suppose that there exists a constant $\hat{\gamma}, 0 \leq \hat{\gamma}<1$, such that there holds either

$$
\beta(x) \geq-\hat{\gamma}, \quad \forall x \in \partial \Omega \cap \overline{\widetilde{C}_{\bar{\varepsilon}}^{ \pm}(\hat{z})},
$$

or

$$
\beta(x) \leq \hat{\gamma}, \quad \forall x \in \partial \Omega \cap \overline{\widetilde{C}_{\bar{\varepsilon}}^{ \pm}(\hat{z})}
$$

Suppose further that for some constant $\mu$ with $\mu \hat{\gamma}<1$ and $C_{\Omega}$ depending only on $\Omega$, an inequality

$$
\int_{\partial \Omega} v d x \leq \mu \int_{\Omega}|D v| d x+C_{\Omega} \int_{\Omega}|v| d x
$$

holds for all $v \in \mathrm{BV}(\Omega)$. If

$$
\left|\tilde{U}_{\varepsilon}^{ \pm}(\hat{z})\right|>0, \quad \forall \varepsilon>0
$$

then there exist positive constants $T_{*}$ and $\alpha_{*}$ determined by $n, \inf _{\Omega \times \mathbb{R}} H, \hat{\gamma}, \mathscr{K}_{\partial \Omega \cap} \overline{\widetilde{C}_{\varepsilon}^{ \pm}(\hat{z})}, R_{0}$, and the geometry of $\Omega$ such that

$$
\frac{d}{d \varepsilon}\left|\tilde{U}_{\varepsilon}^{ \pm}(\hat{z})\right| \geq \alpha_{*} \cdot(n+1) \cdot\left|\tilde{U}_{\varepsilon}^{ \pm}(\hat{z})\right|^{n /(n+1)}
$$

and hence

$$
\left|\tilde{U}_{\varepsilon}^{ \pm}(\hat{z})\right|>\alpha_{*} \varepsilon^{n+1}, \quad \text { for every } \varepsilon \leq T_{*} .
$$


3934 Boundary behavior of capillary surfaces

The value of $\alpha_{*}$ can be specified as

$$
\alpha_{*}=\frac{1-\hat{\gamma}}{16(n+1) k_{(n+1)}},
$$

with $k_{(n+1)}$ being the constant for the isoperimetric inequality in $\mathbb{R}^{n+1}$.

We notice that Giusti in [7, Lemma 1.1] establishes (3.15) for $\mu=1$ in the case that $\partial \Omega \in C^{2}$.

We also notice that an inequality of the form (3.15) appears first by Emmer in [1] with $\mu=\sqrt{1+L^{2}}$ for any Lipschitz domain with Lipschitz constant $L$. By Finn [2, pages 141-143], this result is extended to include domains in which one or more corners with inward opening angle appear.

3.1.2. The result in Theorem 3.1 is connected with the following estimates of $u(x)-$ $u\left(x_{0}\right)$ in terms of the measure of an associated level set.

For $x_{0} \in \bar{\Omega}, T \in \mathbb{R}, T>0$, let us set

$$
\tilde{A}_{T}^{+}\left(x_{0}\right)=\left\{x: x \in \bar{\Omega}, u(x)-u\left(x_{0}\right) \leq T\right\}
$$

and set $\hat{z}_{0}=\left(x_{0}, u\left(x_{0}\right)\right)$. Under the assumption that there exists a constant $\hat{\gamma}, 0 \leq \hat{\gamma}<1$, such that (3.13) holds, by Theorem 3.1 there exist positive constants $T_{*}$ and $\alpha_{*}$ such that

$$
\left|\tilde{U}_{\varepsilon}^{+}\left(\widehat{z}_{0}\right)\right|>\alpha_{*} \varepsilon^{n+1} \quad \text { for every } \varepsilon \leq T_{*} \text {, whenever }\left|\tilde{U}_{\varepsilon}^{+}\left(\widehat{z}_{0}\right)\right|>0 \text { for every } \varepsilon>0 \text {. }
$$

Since $u \in C^{0}(\bar{\Omega})$, we have $\left|\tilde{U}_{T}^{+}\left(\hat{z}_{0}\right)\right| \leq T \cdot\left|\tilde{A}_{T}^{+}\left(x_{0}\right)\right|$, from which and using (3.21), we obtain

$$
T \leq\left(\frac{1}{\alpha_{*}}\left|\tilde{A}_{T}^{+}\left(x_{0}\right)\right|\right)^{1 / n}, \quad \text { for } T \leq T_{*}
$$

Analogously, for $x_{0} \in \bar{\Omega}$ and $T \in \mathbb{R}, T>0$, let us set

$$
\tilde{A}_{T}^{-}\left(x_{0}\right)=\left\{x: x \in \bar{\Omega}, u\left(x_{0}\right)-u(x) \leq T\right\}
$$

Under the assumption that there exists a constant $\hat{\gamma}, 0 \leq \hat{\gamma}<1$, such that (3.14) holds, by Theorem 3.1 there exist positive constants $T_{*}$ and $\alpha_{*}$ such that

$$
\left|\tilde{U}_{\varepsilon}^{-}\left(\hat{z}_{0}\right)\right|>\alpha_{*} \varepsilon^{n+1}, \quad \text { for every } \varepsilon \leq T_{*} \text {, whenever }\left|\tilde{U}_{\varepsilon}^{-}\left(\widehat{z}_{0}\right)\right|>0 \text { for every } \varepsilon>0 \text {, }
$$

holds. We have analogously

$$
T \leq\left(\frac{1}{\alpha_{*}}\left|\tilde{A}_{T}^{-}\left(x_{0}\right)\right|\right)^{1 / n}, \quad \text { for } T \leq T_{*} .
$$


3.2. The Lipschitz continuity of the trace on the boundary: Proof of MainTheorem 1.1. Assume that there exists a nonnegative constant $H_{*}$ such that

$$
|H(x, t)| \leq H_{*}, \quad \text { for } x \in \Omega, t \in \mathbb{R} .
$$

Analogously to [11, Section 4], we restrict our consideration to a small region $\Omega \cap A_{\delta_{0}}\left(x_{0}\right)$ of the type indicated in the beginning of Section 2.4, for some positive number $\delta_{0}$.

Let

$$
\begin{aligned}
& \partial_{-}\left(\Omega \cap A_{\delta_{0}}\left(x_{0}\right)\right)=\left(\partial\left(\Omega \cap A_{\delta_{0}}\left(x_{0}\right)\right) \backslash \partial^{* *} A_{\delta_{0}}\left(x_{0}\right)\right) \cap\left\{x: \beta_{\Omega \cap A_{\delta_{0}}\left(x_{0}\right)}<0\right\} \\
& \partial_{+}\left(\Omega \cap A_{\delta_{0}}\left(x_{0}\right)\right)=\left(\partial\left(\Omega \cap A_{\delta_{0}}\left(x_{0}\right)\right) \backslash \partial^{* *} A_{\delta_{0}}\left(x_{0}\right)\right) \cap\left\{x: \beta_{\Omega \cap A_{\delta_{0}}\left(x_{0}\right)}>0\right\}
\end{aligned}
$$

and let

$$
t_{A}^{+-}=\inf _{\partial_{+}\left(\Omega \cap A_{\delta_{0}}\left(x_{0}\right)\right)} u-u\left(x_{0}\right), \quad t_{A}^{-+}=\sup _{\partial_{-}\left(\Omega \cap A_{\delta_{0}}\left(x_{0}\right)\right)} u-u\left(x_{0}\right)
$$

Let us set

$$
\begin{aligned}
& \left(\Omega \cap A_{\delta_{0}}\left(x_{0}\right)\right)^{+}=\left\{x: x \in \Omega \cap A_{\delta_{0}}\left(x_{0}\right), u(x)-u\left(x_{0}\right) \geq t_{A}^{+-}\right\}, \\
& \left(\Omega \cap A_{\delta_{0}}\left(x_{0}\right)\right)^{-}=\left\{x: x \in \Omega \cap A_{\delta_{0}}\left(x_{0}\right), u(x)-u\left(x_{0}\right) \leq t_{A}^{-+}\right\} .
\end{aligned}
$$

3.2.1. We notice that the following result holds when $\theta \equiv 0$ or $\theta \equiv \pi$ on a portion of $\partial \Omega$. Lemma 3.2. Suppose that $\beta(x) \equiv 1$ or $\beta(x) \equiv-1$ on $\partial \Omega \cap \bar{A}_{\delta_{0}}\left(x_{0}\right)$. For $\delta_{0}$ sufficiently small, $\overline{\left(\Omega \cap A_{\delta_{0}}\left(x_{0}\right)\right)^{+}}$and $\overline{\left(\Omega \cap A_{\delta_{0}}\left(x_{0}\right)\right)^{-}}$are disjoint; in particular, $\partial\left(\left(\Omega \cap A_{\delta_{0}}\left(x_{0}\right)\right)^{ \pm}\right)$does not intersects $\partial_{\mp}\left(\Omega \cap A_{\delta_{0}}\left(x_{0}\right)\right)$.

Proof. Since $u$ is assumed to be bounded up to the boundary, [10, Theorem 1] assures us that $u \in H^{1,1}(\Omega)$. From this and the fact that the restriction $\left.u\right|_{\Omega \backslash \Omega_{\varepsilon}}$ is a minimizing function of the functional

$$
J(u)=\int_{\Omega \backslash \Omega_{\varepsilon}} \sqrt{1+|D u|^{2}} d x+\int_{\partial \Omega_{\varepsilon} \cap \Omega} H u d x+\int_{\partial \Omega_{\varepsilon} \cap \Omega}\left(T u \cdot v_{\Omega_{\varepsilon}}\right) u d \mathcal{H}_{n-1},
$$

with $v_{\Omega_{\varepsilon}}$ being the unit outward normal to $\Omega_{\varepsilon}$, we are allowed to set $\eta=1$ in the identities

$$
\begin{gathered}
\int_{\Omega} \frac{D u}{\sqrt{1+|D u|^{2}}} \cdot D \eta d x+\int_{\Omega} H \cdot \eta d x=\int_{\partial \Omega} \beta \cdot \eta d \mathcal{H}_{n-1}, \\
\int_{\Omega \backslash \Omega_{\varepsilon}} \frac{D u}{\sqrt{1+|D u|^{2}}} \cdot D \eta d x+\int_{\Omega \backslash \Omega_{\varepsilon}} H \cdot \eta d x=\int_{\partial \Omega_{\varepsilon} \backslash \partial \Omega}\left(T u \cdot \nu_{\Omega_{\varepsilon}}\right) \cdot \eta d \mathcal{H}_{n-1} ;
\end{gathered}
$$


here $\Omega_{\varepsilon}=\{x: x \in \Omega$, dist $(x, \partial \Omega) \leq \varepsilon\}$ for sufficiently small $\varepsilon$. Subtracting (3.32) (with $\eta=1$ ) from (3.31) (with $\eta=1$ ), we obtain

$$
\int_{\Omega_{\varepsilon}} H d x=\int_{\partial \Omega} \beta d \mathcal{H}_{n-1}-\int_{\partial \Omega}\left(T u \cdot \nu_{\Omega_{\varepsilon}}\right) d \mathscr{H}_{n-1} \cdot
$$

Since the left-hand side of the last identity approaches zero as $\varepsilon \rightarrow 0$, a subsequence can be extracted from the sequence $\left\{T u \cdot v_{\Omega_{\varepsilon}}(x+D d)\right\}$ with $d(x)=\operatorname{dist}(x, \partial \Omega)$, which approaches $\beta(x)$ for almost every $x \in \partial \Omega$ as $\varepsilon \rightarrow 0$. Under the assumption $|\beta(x)|=1$ on $\partial \Omega \cap A\left(x_{0}\right)$, this, together with the interior regularity of $u$, yields that for each $\delta_{1}$, $0<\delta_{1}<1$, if $\delta_{0}$ is sufficiently small, then

$$
\left|T u \cdot v_{\Omega_{\varepsilon}}\right| \geq \frac{1-\delta_{1}}{2}
$$

for $x \in \partial \Omega_{\varepsilon} \cap \Omega, \varepsilon \leq \delta_{0}$. Thus, if $\beta \equiv 1$ on $\partial \Omega \cap \bar{A}_{\delta_{0}}\left(x_{0}\right)$ and $\delta_{0}$ is sufficiently small, the level set $\left\{x: x \in \Omega \cap A_{\delta_{0}}\left(x_{0}\right), u(x)=t_{A}^{+-}\right\}$intersects $\partial \Omega$ at the angle 0 and stays almost parallel to $\partial \Omega$ and the level set $\left\{x: x \in \Omega \cap A_{\delta_{0}}\left(x_{0}\right), u(x)=t_{A}^{-+}\right\}$intersects $\partial A_{\delta_{0}}\left(x_{0}\right) \cap \Omega$ at the angle 0 and stays almost parallel to $\partial A_{\delta_{0}}\left(x_{0} \cap \Omega\right)$. Thus, if $\beta \equiv-1$ on $\partial \Omega \cap \bar{A}_{\delta_{0}}\left(x_{0}\right)$ and $\delta_{0}$ is sufficiently small, the level set $\left\{x: x \in \Omega \cap A_{\delta_{0}}\left(x_{0}\right), u(x)=t_{A}^{-+}\right\}$intersects $\partial \Omega$ at the angle 0 and stays almost parallel to $\partial \Omega$ and the level set $\left\{x: x \in \Omega \cap A_{\delta_{0}}\left(x_{0}\right), u(x)=t_{A}^{+-}\right\}$ intersects $\partial A_{\delta_{0}}\left(x_{0}\right) \cap \Omega$ at the angle 0 and stays almost parallel to $\partial A_{\delta_{0}}\left(x_{0} \cap \Omega\right)$. From this, follows Lemma 3.2.

3.2.2. Proof of Main Theorem 1.1. Consider a point $x_{0} \in \partial \Omega$ with $\theta\left(x_{0}\right)=0$ and choose $\delta_{0}$ sufficiently small such that $\theta \equiv 0$ on $\partial \Omega \cap B_{\delta_{0}}\left(x_{0}\right)$. Choose the boundary strip $A_{\delta_{0}}\left(x_{0}\right)$ adjacent to $\partial \Omega \cap B_{\delta_{0}}\left(x_{0}\right)$ to be of width $\delta_{0}$ and to be of the type indicated above. By the choice of $A_{\delta_{0}}\left(x_{0}\right)$, we have

$$
\left|\left(\Omega \cap A_{\delta_{0}}\left(x_{0}\right)\right)^{+}\right| \leq\left|\Omega \cap A_{\delta_{0}}\left(x_{0}\right)\right| \leq\left(2 \delta_{0}\right)^{n}
$$

The fact that $\theta \equiv 0$ on $\partial \Omega \cap \overline{A\left(x_{0}\right)}$ and Lemma 3.2 allow us to set $\hat{\gamma}=-1 / 2$ in (3.19) and (3.22) with $T=t_{A}^{+-}$and $\widetilde{A}_{T}^{+}\left(x_{0}\right)=\left(\Omega \cap A_{\delta_{0}}\left(x_{0}\right)\right)^{+}$. Inserting (3.35) into (3.22), we establish Main Theorem 1.1 in case $\theta \equiv \pi$ near $x_{0}$.

In case $\theta \equiv \pi$ in a portion of $\partial \Omega$, consider a point $x_{0} \in \partial \Omega$ with $\theta\left(x_{0}\right)=\pi$ and choose $\delta_{0}$ sufficiently small such that $\theta \equiv \pi$ on $\partial \Omega \cap B_{\delta_{0}}\left(x_{0}\right)$. Choose the boundary strip $A_{\delta_{0}}\left(x_{0}\right)$ adjacent to $\partial \Omega \cap B_{\delta_{0}}\left(x_{0}\right)$ to be of width $\delta_{0}$ and to be of the type indicated above. We have

$$
\left|\left(\Omega \cap A_{\delta_{0}}\left(x_{0}\right)\right)^{-}\right| \leq\left|\Omega \cap A_{\delta_{0}}\left(x_{0}\right)\right| \leq\left(2 \delta_{0}\right)^{n} .
$$

The fact that $\theta \equiv \pi$ on $\partial \Omega \cap \overline{A\left(x_{0}\right)}$ and Lemma 3.2 allow us to set $\hat{\gamma}=1 / 2$ in (3.19) and (3.25) with $T=t_{A}^{-+}$and $\tilde{A}_{T}^{-}\left(x_{0}\right)=\left(\Omega \cap A_{\delta_{0}}\left(x_{0}\right)\right)^{-}$. Inserting (3.36) into (3.25), we establish Main Theorem 1.1 in case $\theta \equiv 0$ near $x_{0}$. 


\section{Proof of Main Theorem 1.2}

Let $u$ be a bounded solution to (1.1) subject to the "contact angle" boundary condition (1.2). Choose a point $x_{0} \in \partial \Omega$. Let $\mathfrak{C}_{x_{0}}$ be the level set of $u$ through $x_{0}$. Since $|\beta|$ is bounded away from 1 , the level set $\mathfrak{C}_{x_{0}}$ divides $\Omega$ into two components $\Omega_{1}$ and $\Omega_{2}$ in which the respective inequalities $u(x) \geq u\left(x_{0}\right)$ and $u(x) \leq u\left(x_{0}\right)$ are valid at points near to $\mathfrak{C}_{x_{0}}$.

We choose, for sufficiently small $t_{0}$, the set $\mathscr{A}_{t_{0}}^{\delta}\left(x_{0}\right)$ for $0 \leq \delta<1$ as follows. Namely, setting $d(x)=\operatorname{dist}\left(x, \mathfrak{C}_{x_{0}}\right)$ for $x \in \Omega$ and letting

$$
\partial^{t} \Omega_{1}=\left\{x: x \in \Omega_{1}, \operatorname{dist}\left(x, \mathfrak{C}_{x_{0}}\right)=t\right\} \quad \text { for } t>0,
$$

we let the boundary $\partial \mathscr{A}_{t_{0}}^{\delta}\left(x_{0}\right)$ be made up of five parts, namely,

$$
\begin{aligned}
\partial \mathscr{A}_{t_{0}}^{\delta}\left(x_{0}\right)= & \left(\partial_{1}^{*} \mathscr{A}_{t_{0}}^{\delta}\left(x_{0}\right)\right) \cup\left(\partial_{2}^{*} \mathscr{A}_{t_{0}}^{\delta}\left(x_{0}\right)\right) \cup\left(\partial_{1}^{* *} \mathscr{A}_{t_{0}}^{\delta}\left(x_{0}\right)\right) \\
& \cup\left(\partial_{2}^{* *} \mathscr{A}_{t_{0}}^{\delta}\left(x_{0}\right)\right) \cup\left(\partial_{3}^{* *} \mathscr{A}_{t_{0}}^{\delta}\left(x_{0}\right)\right),
\end{aligned}
$$

such that

$$
\begin{gathered}
\partial_{1}^{*} \mathscr{A}_{t_{0}}^{\delta}\left(x_{0}\right) \subset \mathfrak{C}_{x_{0}}, \\
\partial_{2}^{*} \mathscr{A}_{t_{0}}^{\delta}\left(x_{0}\right) \subset \partial^{\left(t_{0}\right)^{1-\delta}} \Omega_{1}, \\
\partial_{1}^{* *} \mathscr{A}_{t_{0}}^{\delta}\left(x_{0}\right) \subset \partial \Omega, \\
\partial_{2}^{* *} \mathscr{A}_{t_{0}}^{\delta}\left(x_{0}\right) \subset\left\{x: x \in \Omega, \operatorname{dist}\left(x, \partial_{1}^{* *} \mathscr{A}_{t_{0}}^{\delta}\left(x_{0}\right)\right)=t_{0}\right\}, \\
\operatorname{diam}\left(\partial_{1}^{* *} \mathscr{A}_{t_{0}}^{\delta}\left(x_{0}\right)\right) \leq\left(t_{0}\right)^{1-\delta}, \quad \operatorname{diam}\left(\partial_{1}^{*} \mathscr{A}_{t_{0}}^{\delta}\left(x_{0}\right)\right) \leq t_{0},
\end{gathered}
$$

and $\partial_{1}^{* *} \mathscr{A}_{t_{0}}^{\delta}\left(x_{0}\right), \partial_{1}^{*} \mathscr{A}_{t_{0}}^{\delta}\left(x_{0}\right)$ include $(n-1)$-dimensional balls of the respective radii $\left(t_{0}\right)^{1-\delta / 2}, t_{0} / 2$, and

$$
\begin{aligned}
& \frac{1}{2}\left(t_{0}\right)^{(n-1)(1-\delta)} \leq\left|\partial_{1}^{* *} \mathscr{A}_{t_{0}}^{\delta}\left(x_{0}\right)\right| \leq\left(t_{0}\right)^{(n-1)(1-\delta)}, \\
& \frac{1}{2}\left(t_{0}\right)^{(n-1)} \leq\left|\partial_{1}^{*} \mathscr{A}_{t_{0}}^{\delta}\left(x_{0}\right)\right| \leq\left(t_{0}\right)^{(n-1)} .
\end{aligned}
$$

Furthermore, $\partial_{3}^{* *} \mathscr{A}_{t_{0}}^{\delta}\left(x_{0}\right)$ consists of $c_{n}$ components of $(n-1)$-dimensional surfaces which together with $\partial_{1}^{*} \mathscr{A}_{t_{0}}^{\delta}\left(x_{0}\right), \partial_{2}^{*} \mathscr{A}_{t_{0}}^{\delta}\left(x_{0}\right), \partial_{1}^{* *} \mathscr{A}_{t_{0}}^{\delta}\left(x_{0}\right), \partial_{2}^{* *} \mathscr{A}_{t_{0}}^{\delta}\left(x_{0}\right)$ enclose the connected $n$-dimensional region $\mathscr{A}_{t_{0}}^{\delta}\left(x_{0}\right)$ such that

$$
\left|\partial_{3}^{* *} \mathscr{A}_{t_{0}}^{\delta}\left(x_{0}\right)\right| \leq 2 c_{n}\left(t_{0}\right)^{(n-1)-(n-2) \delta} ;
$$

here $c_{n}$ is a constant depending only on $n$. 
3938 Boundary behavior of capillary surfaces

Let $\eta_{0}$ be a smooth function on $\bar{\Omega}$ such that

$$
\begin{gathered}
0 \leq \eta_{0} \leq 1, \\
\eta_{0}(x)=1 \quad \text { for } x \in \mathscr{A}_{\varepsilon / 2}^{\delta}\left(x_{0}\right), \\
\eta_{0}(x)=0 \quad \text { for } x \in \partial_{2}^{*} \mathscr{A}_{3 \varepsilon / 4}^{\delta}\left(x_{0}\right), \\
4\left(\frac{1}{\varepsilon}\right)^{1-\delta} \leq\left|D \eta_{0}\right| \leq 4 \sqrt{2}\left(\frac{1}{\varepsilon}\right)^{1-\delta}, \quad \text { for } x \in \mathscr{A}_{3 \varepsilon / 4}^{\delta}\left(x_{0}\right) \backslash \mathscr{A}_{\varepsilon / 2}^{\delta}\left(x_{0}\right) .
\end{gathered}
$$

Let $k$ be a number greater than $\max \left(u\left(x_{0}\right), t_{0}, 0\right)$. We set

$$
u_{k}=\left(1-\eta_{0}\right) u+\min \left(\eta_{0} u, k\right)
$$

Then, $u_{k}$ belongs to $\mathrm{BV}(\Omega)$ and from the minimizing property of $u$, we know that setting

$$
\mathscr{A}_{0}\left(k, \eta_{0}\right)=\left\{x: x \in \mathscr{A}_{3 \varepsilon / 4}^{\delta}\left(x_{0}\right) \cap \bar{\Omega}, \eta_{0} u>k\right\}
$$

the restriction $\left.u\right|_{\mathscr{A}_{0}\left(k, \eta_{0}\right)}$ is a capillary surface such that

$$
J_{k}(u) \leq J_{k}\left(u_{k}\right)
$$

where for $v \in C^{1}(\Omega)$, we set

$$
\begin{aligned}
J_{k}(v)=\int_{\mathscr{A}_{0}\left(k, \eta_{0}\right)} \sqrt{1+|D v|^{2}} d x+\int_{\mathscr{A}_{0}\left(k, \eta_{0}\right)} \int_{0}^{v} H(x, t) d t d x \\
\quad+\int_{\partial \mathscr{A}_{0}\left(k, \eta_{0}\right) \backslash \partial_{1}^{* *} \mathscr{A}_{\mathcal{H}_{\varepsilon / 4}}^{\delta}\left(x_{0}\right)}\left(T v \cdot v_{\mathscr{A}_{0}\left(k, \eta_{0}\right)}\right) v d \mathscr{H}_{n-1}+\int_{\partial \mathscr{A}_{0}\left(k, \eta_{0}\right) \cap \partial_{1}^{* *} \mathscr{A}_{\mathcal{H}_{\varepsilon / 4}}^{\delta}\left(x_{0}\right)} \beta v d \mathcal{H}_{n-1},
\end{aligned}
$$

where $\nu_{\mathscr{A}_{0}\left(k, \eta_{0}\right)}$ is the unit outward normal to $\mathscr{A}_{0}\left(k, \eta_{0}\right)$ and we set $(f)^{-}=\min (f, 0)$ for all real-valued functions $f$. Assume for a moment that $u$ is smooth, we obtain analogously to $[9$, equation $(1.8)]$ that

$$
\begin{aligned}
& \int_{\mathscr{A}_{0}\left(k, \eta_{0}\right)} \sqrt{1+|D u|^{2}} d x+\int_{\mathscr{A}_{0}\left(k, \eta_{0}\right)} \int_{u_{k}}^{u} H(x, t) d t d x \\
& \leq \int_{\mathscr{A}_{0}\left(k, \eta_{0}\right)} \sqrt{1+\left|D\left[\left(1-\eta_{0}\right) u\right]\right|^{2}} d x+\int_{\overline{\mathscr{A}_{0}\left(k, \eta_{0}\right)} \cap \partial_{1}^{*} \mathscr{A}_{\xi \varepsilon / 4}^{\delta}\left(x_{0}\right)} \beta\left(\eta_{0} u-k\right) d \mathscr{H}_{n-1} \\
& +\int_{\overline{A_{0}\left(k, \eta_{0}\right)} \cap \partial_{0}^{* *} \mathscr{A}_{\mathcal{E} / 4}^{\delta}\left(x_{0}\right)} \beta_{1}(\eta u-k) d \mathscr{H}_{n-1}-\int_{\partial \mathscr{A}_{0}\left(k, \eta_{0}\right)} u \frac{\left(D \eta_{0} \cdot \nu_{0}\right)^{-}}{\sqrt{1+u^{2}\left|D \eta_{0}\right|^{2}}} d \mathscr{H}_{n-1}
\end{aligned}
$$




$$
\begin{aligned}
& \leq \int_{\mathscr{A}_{0}\left(k, \eta_{0}\right)} \sqrt{1+\left(1-\eta_{0}\right)^{2}|D u|^{2}} d x+\int_{\mathscr{A}_{0}\left(k, \eta_{0}\right)} \sqrt{1+u^{2}\left|D \eta_{0}\right|^{2}} d x \\
& -\int_{\partial \mathscr{A}_{0}\left(k, \eta_{0}\right)} u \frac{\left(D \eta_{0} \cdot \nu_{0}\right)^{-}}{\sqrt{1+u^{2}\left|D \eta_{0}\right|^{2}}} d \mathscr{H}_{n-1} \\
& +\int_{\overline{\mathcal{A}_{0}\left(k, \eta_{0}\right)} \cap \partial_{0}^{* *} \mathscr{A}_{3 \varepsilon / 4}^{\delta}\left(x_{0}\right)} \beta_{1}\left(\eta_{0}-1\right)\left(\eta_{0} u-k\right) d \mathcal{H}_{n-1} \\
& +\int_{\overline{\mathbb{A}_{0}\left(k, \eta_{0}\right)} \cap \partial_{1}^{*} \mathscr{A}_{3 \varepsilon / 4}^{\delta}\left(x_{0}\right)} \beta\left(\eta_{0}-1\right)\left(\eta_{0} u-k\right) d \mathscr{H}_{n-1},
\end{aligned}
$$

where we set $\partial_{0}^{* *} \mathscr{A}_{3 \varepsilon / 4}^{\delta}\left(x_{0}\right)=\partial_{3}^{* *} \mathscr{A}_{3 \varepsilon / 4}^{\delta}\left(x_{0}\right) \cup \partial_{2}^{* *} \mathscr{A}_{3 \varepsilon / 4}^{\delta}\left(x_{0}\right)$ and $\beta_{1}=T u \cdot \nu_{\mathscr{A}_{3 \varepsilon / 4}^{\delta}\left(x_{0}\right)}$, with $v_{\mathscr{A}_{3 \varepsilon / 4}^{\delta}\left(x_{0}\right)}$ being the unit outward normal to $\mathscr{A}_{3 \varepsilon / 4}^{\delta}\left(x_{0}\right)$ and $\nu_{0}$ is the unit outward normal to $\mathscr{A}_{0}\left(k, \eta_{0}\right)$.

Setting $w=\max \left(\eta_{0} u-k, 0\right)$, this and the monotonicity condition on $H$ in (1.4) yield, analogously to [9, equation (1.10)],

$$
\begin{aligned}
& \int_{\mathscr{A}_{0}\left(k, \eta_{0}\right)}|D w| d x+\int_{\mathscr{A}_{0}\left(k, \eta_{0}\right)} H_{t_{0}} w d x \\
& \leq 2\left|\mathscr{A}_{0}\left(k, \eta_{0}\right)\right|+2\left(\sup _{\mathscr{A}_{3 \varepsilon / 4}^{\delta}\left(x_{0}\right)}\left|D \eta_{0}\right|\right) \int_{\mathscr{A}_{0}\left(k, \eta_{0}\right)} u d x-\int_{\partial \mathscr{A}_{0}\left(k, \eta_{0}\right)} u \frac{\left(D \eta_{0} \cdot v_{0}\right)^{-}}{\sqrt{1+u^{2}\left|D \eta_{0}\right|^{2}}} d \mathscr{H}_{n-1} \\
& +\int_{\overline{\mathscr{A}_{0}\left(k, \eta_{0}\right)} \cap \partial_{0}^{* *} \mathscr{A}_{3 \varepsilon / 4}^{\delta}\left(x_{0}\right)}\left|\beta_{1}\right| w d \mathcal{H}_{n-1}+\int_{\overline{\mathscr{A}_{0}\left(k, \eta_{0}\right)} \cap \partial_{1}^{*} \mathscr{A}_{\mathcal{B}_{\varepsilon / 4}}^{\delta}\left(x_{0}\right)}|\beta| w d \mathcal{H}_{n-1} \text {, }
\end{aligned}
$$

where $H_{t_{0}}=H\left(x, t_{0}\right)$. From the modified version of Sobolev inequality in [12, Theorem 6.5.7], the monotonicity condition on $H$ in (1.4), the condition (1.10) and Hölder's inequality, we obtain

$$
\begin{aligned}
{\left[\left(\frac{\omega_{n}}{n}\right)\right.} & \left.-\left.|| H_{t_{0}}\right|_{L^{p}(\Omega)} \cdot\left|\mathscr{A}_{0}\left(k, \eta_{0}\right)\right|^{(p-n) / n p}\right] \cdot\|w\|_{L_{*}^{n}\left(\mathscr{A}_{0}\left(k, \eta_{0}\right)\right)} \\
\leq & 2\left|\mathscr{A}_{0}\left(k, \eta_{0}\right)\right|+2\left(\sup _{\mathscr{A}_{3 \varepsilon / 4}^{\delta}\left(x_{0}\right)}\left|D \eta_{0}\right|\right) \cdot \int_{\mathscr{A}_{0}\left(k, \eta_{0}\right)} u d x \\
& -2 \int_{\partial \mathscr{A}_{0}\left(k, \eta_{0}\right)} u\left(\frac{D \eta_{0} \cdot \nu_{0}}{\sqrt{1+u^{2}\left|D \eta_{0}\right|^{2}}}\right)^{-} d \mathscr{H}_{n-1} \\
& +2 \int_{\overline{\mathcal{A}_{0}\left(k, \eta_{0}\right)} \cap \partial_{0}^{* *} \mathscr{A}_{\xi \varepsilon / 4}^{\delta}\left(x_{0}\right)}\left|\beta_{1}\right|\left(\eta_{0} u-k\right) d \mathscr{H}_{n-1} \\
& +\int_{\overline{\mathscr{A}_{0}\left(k, \eta_{0}\right)} \cap \partial_{1}^{* * *} \mathscr{A}_{3 \varepsilon / 4}^{\delta}\left(x_{0}\right)}|\beta|\left(\eta_{0} u-k\right) d \mathscr{H}_{n-1}
\end{aligned}
$$


3940 Boundary behavior of capillary surfaces

for $n_{*}=n /(n-1)$ and then, analogously to [9, equation (1.11)], we have

$$
\begin{aligned}
& (h-k) \cdot\left|\mathscr{A}_{0}\left(h, \eta_{0}\right)\right| \\
& \leq 2 C_{0}\left|\mathscr{A}_{0}\left(k, \eta_{0}\right)\right|^{1+1 / n}+2 C_{0}\left(\sup _{\mathscr{A}_{\xi_{\varepsilon / 4}}^{\delta}\left(x_{0}\right)}\left|D \eta_{0}\right|\right) \cdot\left|\mathscr{A}_{0}\left(k, \eta_{0}\right)\right|^{1 / n} \cdot \int_{\mathscr{A}_{0}\left(k, \eta_{0}\right)} u d x \\
& -2 C_{0} \cdot\left|\mathscr{A}_{0}\left(k, \eta_{0}\right)\right|^{1 / n} \cdot \int_{\partial \mathscr{A}_{0}\left(k, \eta_{0}\right)} u\left(\frac{D \eta_{0} \cdot v_{0}}{\sqrt{1+u^{2}\left|D \eta_{0}\right|^{2}}}\right)^{-} d \mathscr{H}_{n-1}
\end{aligned}
$$

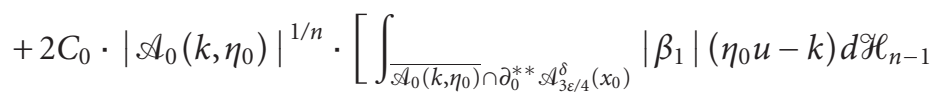

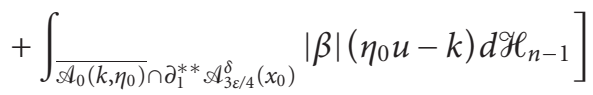

for each $h>k>\max \left(u\left(x_{0}\right), t_{0}, 0\right)$, where

$$
C_{0}=2\left(\frac{n}{\omega_{n}}\right) \leq\left[\left(\frac{\omega_{n}}{n}\right)-\left\|H_{t_{0}}\right\|_{L^{p}(\Omega)} \cdot\left|\mathscr{A}_{3 \varepsilon / 4}^{\delta}\left(x_{0}\right)\right|^{(p-n) / n p}\right]^{-1}
$$

with $\varepsilon$ being so small that

$$
\left\|H_{t_{0}}\right\|_{L^{p}(\Omega)} \cdot\left|\mathscr{A}_{3 \varepsilon / 4}^{\delta}\left(x_{0}\right)\right|^{(p-n) / n p} \leq \frac{1}{2}\left(\frac{\omega_{n}}{n}\right) .
$$

Setting, for $i \in \mathbb{N} \cup\{0\}, \varepsilon_{i}=\sum_{1}^{i+2}\left(\varepsilon / 2^{i}\right)$, and

$$
\begin{gathered}
0 \leq \eta_{i} \leq 1, \\
\eta_{i}(x)=1 \quad \text { for } x \in \mathscr{A}_{\varepsilon_{i-1}}^{\delta}\left(x_{0}\right), \\
\eta_{i}(x)=0 \quad \text { for } x \in \partial_{2}^{*} \mathscr{A}_{\varepsilon_{i}}^{\delta}\left(x_{0}\right), \\
2^{i+2}\left(\frac{1}{\varepsilon}\right)^{1-\delta} \leq\left|D \eta_{0}\right| \leq 2^{i+2} \sqrt{2}\left(\frac{1}{\varepsilon}\right)^{1-\delta}, \quad \text { for } x \in \mathscr{A}_{\varepsilon_{i}}^{\delta}\left(x_{0}\right) \backslash \mathscr{A}_{\varepsilon_{i-1}}^{\delta}\left(x_{0}\right) .
\end{gathered}
$$

and letting

$$
\mathscr{A}_{i}\left(k, \eta_{i}\right)=\left\{x: x \in A_{\varepsilon_{i}}\left(x_{0}\right), \eta_{i} u>k\right\}
$$


we can, analogously to [9, equation (1.13)], derive the following inequality in case $\varepsilon$ is so small that (4.16) and (4.5) hold, namely,

$$
\begin{aligned}
& (h-k) \cdot\left|\mathscr{A}_{i}(h, \eta)\right| \\
& \leq 2 C_{0}\left|\mathscr{A}_{i}\left(k, \eta_{i}\right)\right|^{1+1 / n}+2 C_{0}\left(\sup _{\mathscr{A}_{\varepsilon_{i}}^{\delta_{i}}\left(x_{0}\right)}\left|D \eta_{i}\right|\right) \cdot\left|\mathscr{A}_{i}\left(k, \eta_{i}\right)\right|^{1 / n} \cdot \int_{\mathscr{A}_{i}\left(k, \eta_{i}\right)} u d x \\
& -2 C_{0} \cdot\left|\mathscr{A}_{i}\left(k, \eta_{i}\right)\right|^{1 / n} \cdot \int_{\partial \mathscr{A}_{i}\left(k, \eta_{i}\right)} u \frac{\left(D \eta_{i} \cdot v_{i}\right)^{-}}{\sqrt{1+\left|D \eta_{i}\right|^{2}}} d \mathscr{H}_{n-1} \\
& +2 C_{0} \cdot\left|\mathscr{A}_{i}\left(k, \eta_{i}\right)\right|^{1 / n} \cdot\left[\int_{\overline{A_{i}\left(k, \eta_{i}\right)} \cap \partial_{0}^{* *} A_{\varepsilon_{i}}\left(x_{0}\right)}\left|\beta_{i-1}\right|\left(\eta_{i} u-k\right) d \mathscr{H}_{n-1}\right. \\
& \left.+\int_{\overline{A_{i}\left(k, \eta_{i}\right)} \cap \partial_{1}^{* *} A_{\varepsilon_{i}}\left(x_{0}\right)}|\beta|\left(\eta_{i} u-k\right) d \mathscr{H}_{n-1}\right],
\end{aligned}
$$

where $\partial_{0}^{* *} A_{\varepsilon_{i}}\left(x_{0}\right)=\partial_{2}^{* *} A_{\mathcal{\varepsilon}_{i}}\left(x_{0}\right) \cup \partial_{3}^{* *} A_{\mathcal{\varepsilon}_{i}}\left(x_{0}\right)$ and $\beta_{i-1}=T u \cdot \nu_{A_{\varepsilon_{i}}\left(x_{0}\right)}$, with $\nu_{A_{\varepsilon_{i}}}$ being the unit outward normal to $A_{\varepsilon_{i}}\left(x_{0}\right)$ and $\nu_{i}$ is the unit outward normal to $\mathscr{A}_{\varepsilon_{i-1}}^{\delta}\left(x_{0}\right)$.

4.1. We will apply Lemma 2.3 stated in Section 2.3. We will adapt the iteration procedure on [4, pages 178-179] or [9] to increase the exponent in (4.19) so that (2.12) can be applied. Namely, we firstly let $(u \eta)^{+}=\max (u \eta, 0)$ and conclude by (4.14) and (2.11) that

$$
\left(u \eta_{1}\right)^{+} \in L^{q_{0}}\left(\mathscr{A}_{7 \varepsilon / 8}^{\delta}\left(x_{0}\right)\right)
$$

for any $q_{0}, 1<q_{0}<n /(n-1)$ and that

$$
\begin{aligned}
& \left\|\left(u \eta_{1}\right)^{+}\right\|_{q_{0}} \leq 2^{q_{0}+1} \cdot\left(C_{0} \cdot\left(\sup _{\mathscr{A}_{7 \varepsilon / 8}^{\delta}\left(x_{0}\right)}\left|D \eta_{1}\right|\right) \cdot \int_{\mathscr{A}_{1}\left(k, \eta_{1}\right)} u d x\right.
\end{aligned}
$$

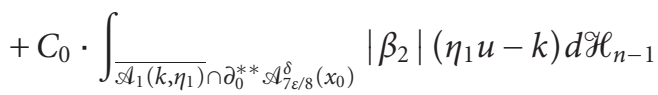

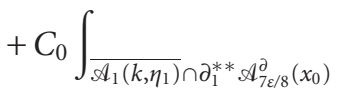

$$
\begin{aligned}
& -C_{0} \int_{\partial \mathscr{A}_{1}\left(k, \eta_{1}\right)} u \frac{\left(D \eta_{1} \cdot v_{1}\right)^{-}}{\sqrt{1+u^{2}\left|D \eta_{1}\right|^{2}}} d \mathscr{H}_{n-1} \\
& \left.+C_{0} \cdot\left|\mathscr{A}_{1}\left(k, \eta_{1}\right)\right|+\max \left(u\left(x_{0}\right), t_{0}, 0\right) \cdot\left|\mathscr{A}_{1}\left(k, \eta_{1}\right)\right|^{1 / q_{0}}\right) \text {, }
\end{aligned}
$$


3942 Boundary behavior of capillary surfaces

where $\partial_{0}^{* *} \mathscr{A}_{7 \varepsilon / 8}^{\delta}\left(x_{0}\right)=\partial_{2}^{* *} \mathscr{A}_{7 \varepsilon / 8}^{\delta}\left(x_{0}\right) \cup \partial_{3}^{* *} \mathscr{A}_{7 \varepsilon / 8}^{\delta}\left(x_{0}\right)$. Since $\eta_{1}=1$ in $\mathscr{A}_{0}\left(k, \eta_{0}\right) \subset$ $\mathscr{A}_{3 \varepsilon / 4}^{\delta}\left(x_{0}\right)$, we obtain from Hölder's inequality that

$$
\int_{\mathscr{A}_{0}\left(k, \eta_{0}\right)} u d x \leq\left\|\left(u \eta_{1}\right)^{+}\right\|_{q_{0}} \cdot\left|\mathscr{A}_{0}\left(k, \eta_{0}\right)\right|^{1-1 / q_{0}} .
$$

Inserting the previous two inequalities into (4.14), we obtain, analogously to [9, equation (1.15)],

$$
\begin{aligned}
& (h-k) \cdot\left|\mathscr{A}_{0}\left(h, \eta_{0}\right)\right| \\
& \leq 2 C_{0} \cdot\left|\mathscr{A}_{0}\left(k, \eta_{0}\right)\right|^{1+1 / n}+2 C_{0} \cdot\left(\sup _{\mathscr{A}_{3 \varepsilon / 4}^{\delta}\left(x_{0}\right)}\left|D \eta_{0}\right|\right) \\
& \cdot\left\{C_{0} \cdot\left(\sup _{\mathscr{A}_{7 \varepsilon / 8}^{\delta}\left(x_{0}\right)}^{\delta}\left|D \eta_{1}\right|\right) \cdot \int_{\mathscr{A}_{1}\left(k, \eta_{1}\right)} u d x\right. \\
& +C_{0} \int_{\overline{\mathcal{A}_{1}\left(k, \eta_{1}\right)} \cap \partial_{0}^{* *} \mathscr{A}_{\overline{7 \varepsilon} / 8}^{\delta}\left(x_{0}\right)}\left|\beta_{2}\right|\left(\eta_{1} u-k\right) d \mathscr{H}_{n-1} \\
& +C_{0} \int_{\overline{A_{1}\left(k, \eta_{1}\right)} \cap \partial_{1}^{* *} \mathscr{A}_{\overline{7 \varepsilon} / 8}^{\partial}\left(x_{0}\right)} \\
& -C_{0} \int_{\partial \mathscr{A}_{1}\left(k, \eta_{1}\right)} u \frac{\left(D \eta_{1} \cdot \nu_{1}\right)^{-}}{\sqrt{1+u^{2}\left|D \eta_{1}\right|^{2}}} d \mathscr{H}_{n-1}+C_{0} \cdot\left|\mathscr{A}_{1}\left(k, \eta_{1}\right)\right| \\
& \left.+\max \left(u\left(x_{0}\right), t_{0}, 0\right) \cdot\left|\mathscr{A}_{1}\left(k, \eta_{1}\right)\right|^{1 / q_{0}}\right\} \cdot\left|\mathscr{A}_{0}\left(k, \eta_{0}\right)\right|^{1-\left(1 / q_{0}\right)+(1 / n)} \\
& +2 C_{0}\left|\mathscr{A}_{0}\left(k, \eta_{0}\right)\right|^{1 / n}\left[\int_{\overline{\mathcal{A}_{0}\left(k, \eta_{0}\right)} \cap \partial_{0}^{* *} \mathscr{A}_{\xi \varepsilon / 4}^{\delta}\left(x_{0}\right)}\left|\beta_{1}\right|\left(\eta_{0} u-k\right) d \mathscr{H}_{n-1}\right. \\
& +\int_{\overline{\mathscr{A}_{0}\left(k, \eta_{0}\right)} \cap \partial_{1}^{* *} \mathscr{A}_{\mathcal{H}_{\varepsilon / 4}}^{\delta}\left(x_{0}\right)}|\beta|\left(\eta_{0} u-k\right) d \mathscr{H}_{n-1} \\
& \left.-C_{0} \int_{\partial \mathscr{A}_{0}\left(k, \eta_{0}\right)} u\left(\frac{\left(D \eta_{0} \cdot \nu_{0}\right)^{-}}{\sqrt{1+u^{2}\left|D \eta_{0}\right|^{2}}}\right) d \mathscr{H}_{n-1}\right] \text {. }
\end{aligned}
$$

Setting $\left(u \eta_{2}\right)^{+}=\max \left(u \eta_{2}, 0\right)$, we derive, analogously to [9, equation (1.16.1)],

$$
\left(u \eta_{2}\right)^{+} \in L^{q_{1}}\left(\mathscr{A}_{15 \varepsilon / 16}^{\delta}\left(x_{0}\right)\right), \quad \text { for any } q_{1}, 1<q_{1}<\frac{n}{n-2},
$$


and also

$$
\begin{aligned}
& (h-k) \cdot\left|\mathscr{A}_{0}\left(h, \eta_{0}\right)\right| \leq 2 C_{0} \cdot\left|\mathscr{A}_{0}\left(k, \eta_{0}\right)\right|^{1+1 / n}+2^{q_{0}+q_{1}+2} C_{0} \cdot\left(\sup _{\mathscr{A}_{3 \varepsilon / 4}^{\delta}\left(x_{0}\right)}\left|D \eta_{0}\right|\right) \\
& \times\left\{( \operatorname { s u p } _ { \mathscr { A } _ { 1 5 \varepsilon / 1 6 } ^ { \delta } ( x _ { 0 } ) } | D \eta _ { 2 } | ) \cdot \left[C_{0} \cdot\left(\sup _{\mathscr{A}_{31 \varepsilon / 32}^{\delta}\left(x_{0}\right)}\left|D \eta_{3}\right|\right) \cdot \int_{\mathscr{A}_{2}\left(k, \eta_{2}\right)} u d x\right.\right. \\
& +C_{0} \cdot \int_{\overline{\mathcal{A}_{3}\left(k, \eta_{3}\right)} \cap \partial_{0}^{* *} \mathscr{A}_{31 \varepsilon / 32}^{\delta}\left(x_{0}\right)}\left|\beta_{4}\right|\left(\eta_{3} u-k\right) d \mathscr{H}_{n-1} \\
& +C_{0} \int_{\overline{A_{3}\left(k, \eta_{3}\right)} \cap \partial_{1}^{* *} \mathscr{A}_{31 \varepsilon / 22}^{\delta}\left(x_{0}\right)}|\beta|\left(\eta_{3} u-k\right) d \mathscr{H}_{n-1} \\
& -C_{0} \int_{\partial \mathscr{A}_{3}\left(k, \eta_{3}\right)} u \frac{\left(D \eta_{3} \cdot v_{3}\right)^{-}}{\sqrt{1+u^{2}\left|D \eta_{3}\right|^{2}}} d \mathscr{H}_{n-1} \\
& \left.+C_{0} \cdot\left|\mathscr{A}_{3}\left(k, \eta_{3}\right)\right|+\max \left(u\left(x_{0}\right), t_{0}, 0\right) \cdot\left|\mathscr{A}_{3}\left(k, \eta_{3}\right)\right|^{1 / q_{0}}\right] \\
& +C_{0}\left|\mathscr{A}_{2}\left(k, \eta_{2}\right)\right|^{1 / q_{0}-1} \cdot\left[\int_{\overline{\mathscr{A}_{2}\left(k, \eta_{2}\right)} \cap \partial_{0}^{* *} \mathscr{A}_{15 \varepsilon / 16}^{\partial}\left(x_{0}\right)}\left|\beta_{3}\right|\left(\eta_{2} u-k\right) d \mathscr{H}_{n-1}\right. \\
& +\int_{\overline{A_{2}\left(k, \eta_{2}\right)} \cap \partial_{1}^{* *} \mathscr{A}_{15 \varepsilon / 16}^{\delta}\left(x_{0}\right)}|\beta|\left(\eta_{2} u-k\right) d \mathscr{H}_{n-1} \\
& \left.-C_{0} \int_{\partial \mathscr{A}_{2}\left(k, \eta_{2}\right)} u \frac{\left(D \eta_{2} \cdot v_{2}\right)^{-}}{\sqrt{1+u^{2}\left|D \eta_{2}\right|^{2}}} d \mathscr{H}_{n-1}\right] \\
& +C_{0} \cdot\left|\mathscr{A}_{2}\left(k, \eta_{2}\right)\right|^{1 / q_{0}} \\
& \left.+\max \left(u\left(x_{0}\right), t_{0}, 0\right) \cdot\left|\mathscr{A}_{2}\left(k, \eta_{2}\right)\right|^{1 / q_{1}}\right\} \cdot\left|\mathscr{A}_{0}\left(k, \eta_{0}\right)\right|^{1-\left(1 / q_{1}\right)+(1 / n)} \\
& +C_{0}\left|\mathscr{A}_{0}\left(k, \eta_{0}\right)\right|^{1 / n}\left[\int_{\overline{\mathcal{A}_{0}\left(k, \eta_{0}\right)} \cap \partial_{0}^{* *} \mathscr{A}_{3 \varepsilon / 4}^{\delta}\left(x_{0}\right)}\left|\beta_{1}\right|\left(\eta_{0} u-k\right) d \mathscr{H}_{n-1}\right. \\
& +\int_{\overline{\mathscr{A}_{0}\left(k, \eta_{0}\right)} \cap \partial_{1}^{* *} \mathscr{A}_{3 \varepsilon / 4}^{\delta}\left(x_{0}\right)}|\beta|\left(\eta_{0} u-k\right) d \mathscr{H}_{n-1} \\
& \left.-C_{0} \int_{\partial \mathscr{A}_{0}\left(k, \eta_{0}\right)} u \frac{\left(D \eta_{0} \cdot \nu_{0}\right)^{-}}{\sqrt{1+u^{2}\left|D \eta_{0}\right|^{2}}} d \mathscr{H}_{n-1}\right] \text {, }
\end{aligned}
$$

for each $h>k>\max \left(u\left(x_{0}\right), t_{0}, 0\right)$ and $1<q_{1}<n /(n-2)$. 
4.2. We continue the process of iteration so that the exponent of $\left|\mathscr{A}_{0}\left(k, \eta_{0}\right)\right|$ is increased by a number $1 /\left(q_{m}-1\right)-1 / q_{m}$ at each step,

$$
1<q_{m}<\left[1-\frac{1}{n}-\left(1-\frac{1}{q_{m-1}}\right)\right]^{-1}<\frac{n}{n-(m+1)},
$$

and $q_{m}$ can be arbitrarily close to $n /(n-(m+1))$. After $n_{0}+1$ steps, $1 \leq n_{0} \leq n-1$, the exponent of $\left|\mathscr{A}_{0}\left(k, \eta_{0}\right)\right|$ increased to become greater than 1 . That is, setting, for $1 \leq m \leq$ $n_{0}$,

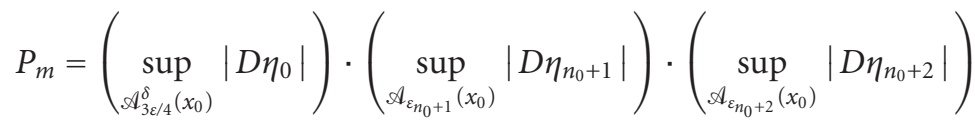

$$
\begin{aligned}
& \cdots\left(\sup _{A_{\varepsilon_{n_{0}+m}\left(x_{0}\right)}}\left|D \eta_{n_{0}+m}\right|\right) \cdot\left(\sup _{\mathscr{A}_{\varepsilon_{n_{0}+m+1}}\left(x_{0}\right)}\left|D \eta_{n_{0}+m+1}\right|\right) \text {, } \\
& P_{0}=\left(\sup _{\mathscr{A}_{3 \varepsilon / 4}^{\delta}\left(x_{0}\right)}\left|D \eta_{0}\right|\right), \quad P_{-1}=1,
\end{aligned}
$$

and letting

$$
\begin{aligned}
\widetilde{C}=\sum_{m=-1}^{n_{0}-1}\left(C_{0}\right)^{m} P_{m}( & C_{0}\left|\mathscr{A}_{n_{0}+m+1}\left(k, \eta_{n_{0}+m+1}\right)\right|^{1 / q_{n_{0}-m-2}} \\
& \left.+\max \left(u\left(x_{0}\right), t_{0}, 0\right)\left|\mathscr{A}_{n_{0}+m+1}\left(k, \eta_{n_{0}+m+1}\right)\right|^{1 / q_{n_{0}-m-1}}\right),
\end{aligned}
$$

with $q_{-1}=1$, we finally arrive at, analogously to [9, equation (1.19)],

$$
\begin{aligned}
&(h-k) \cdot \mid\left.\mathscr{A}_{0}\left(h, \eta_{0}\right)|\leq 2| \mathscr{A}_{0}\left(k, \eta_{0}\right)\right|^{1+1 / n}+2^{\hat{C}_{n_{0}}}\left|\mathscr{A}_{0}\left(k, \eta_{0}\right)\right|^{\left(1-\left(1 / q_{n_{0}}\right)\right)+(1 / n)} \\
& \cdot {\left[\left(C_{0}\right)^{n_{0}} P_{n_{0}} \int_{\mathscr{A}_{2 n_{0}-1}\left(k, \eta_{2 n_{0}-1}\right)} u d x+\sum_{m=-1}^{n_{0}-1}\left(C_{0}\right)^{m} P_{m} \cdot\left|\mathscr{A}_{n_{0}+m}\left(k, \eta_{n_{0}+m}\right)\right|^{1 / q_{n_{0}-m-2}-1}\right.} \\
& \cdot\left(\int_{\overline{A_{n_{0}+m}\left(k, \eta_{n_{0}+m}\right)} \cap \partial_{0}^{* *} \mathscr{A}_{\varepsilon_{m}}\left(x_{0}\right)}\left|\beta_{m+1}\right|\left(\eta_{n_{0}+m} u-k\right) d \mathscr{H}_{n-1}\right. \\
& \quad+\int_{\mathscr{A}_{n_{0}+m}\left(k, \eta_{n_{0}+m}\right)} \cap \partial_{1}^{* *} \mathscr{A}_{\varepsilon_{n_{0}+m}\left(x_{0}\right)}|\beta|\left(\eta_{n_{0}+m} u-k\right) d \mathscr{H}_{n-1} \\
&\left.\left.\quad-C_{0} \int_{\partial \mathscr{A}_{n_{0}+m}\left(k, \eta_{n_{0}+m}\right)} u \frac{\left(D \eta_{n_{0}+m} \cdot v_{n_{0}+m}\left(x_{0}\right)\right)^{-}}{\sqrt{1+u^{2}\left|D \eta_{n_{0}+m}\right|^{2}}} d \mathscr{H}_{n-1}\right)+\tilde{C}\right]
\end{aligned}
$$




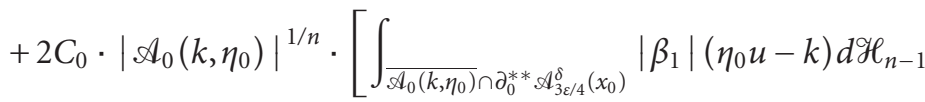

$$
\begin{aligned}
& +\int_{\overline{\mathcal{A}_{0}\left(k, \eta_{0}\right)} \cap \partial_{1}^{* *} \mathscr{A}_{\mathcal{B}_{\varepsilon / 4}}^{\delta}\left(x_{0}\right)}|\beta|\left(\eta_{0} u-k\right) d \mathscr{H}_{n-1} \\
& \left.-C_{0} \int_{\partial \mathscr{A}_{0}\left(k, \eta_{0}\right)} u \frac{\left(D \eta_{0} \cdot \nu_{0}\right)^{-}}{\sqrt{1+u^{2}\left|D \eta_{0}\right|^{2}}} d \mathscr{H}_{n-1}\right] \text {, }
\end{aligned}
$$

for each $h>k>\max \left(u\left(x_{0}\right), t_{0}, 0\right)$, where

$$
\left(1-\left(\frac{1}{q_{n_{0}}}\right)\right)+\frac{1}{n} \geq 1 \quad \text { for } 1 \leq m \leq n_{0},
$$

and $\varepsilon_{n_{0}}=\sum_{i=1}^{n_{0}}\left(\varepsilon / 2^{i}\right)$ and $\widehat{C}_{n_{0}}=2^{q_{0}+q_{1}+\cdots+q_{n_{0}-1}+q_{n_{0}}}$. We may assume without loss of generality that

$$
u\left(x_{0}\right)=0, \quad t_{0} \leq 0 .
$$

Thus, setting $\widetilde{C}_{0}=2+\left((1 / n)+\left(1 / q_{n_{0}}\right)\right)^{-1}+\widehat{C}_{0}$, we obtain from this, (4.6), and (2.12) that

$$
\begin{aligned}
& \sup u-u\left(x_{0}\right) \leq 2^{\widetilde{C}_{0}} C_{0} \cdot\left|\mathscr{A}_{0}\left(k, \eta_{0}\right)\right|^{1 / n}+2^{\widetilde{C}_{0}} \widetilde{C} \cdot\left|\mathscr{A}_{0}\left(k, \eta_{0}\right)\right|^{(1 / n)-\left(1 / q_{n_{0}}\right)} \\
& \mathscr{A}_{\varepsilon / 2}^{\delta}\left(x_{0}\right) \\
& +2 \widetilde{C}^{\widetilde{C}_{0}} C_{0}\left(\int_{\partial \mathscr{A}_{0}\left(k, \eta_{0}\right)} u \frac{\left(D \eta_{0} \cdot v_{0}\right)^{-}}{\sqrt{1+u^{2}\left|D \eta_{0}\right|^{2}}} d \mathscr{H}_{n-1}\right)\left|\mathscr{A}_{0}\left(k, \eta_{0}\right)\right|^{1 / n-1} \\
& +2^{\widetilde{C}_{0}} \cdot\left(C_{0}\right)^{n_{0}} \cdot P_{n_{0}} \cdot\left(\sup _{\mathscr{A}_{\varepsilon}^{\delta}\left(x_{0}\right)} u-\inf _{\mathscr{A}_{\varepsilon}^{\delta}\left(x_{0}\right)} u\right) \cdot\left|\mathscr{A}_{0}\left(k, \eta_{0}\right)\right|^{1-\left(1 / q_{n_{0}}\right)+(1 / n)} \\
& +2^{\widetilde{C}_{0}} \cdot \sum_{m=-1}^{n_{0}-1}\left(C_{0}\right)^{m} P_{m} \cdot\left(\sup _{\mathscr{A}_{\varepsilon}^{\delta}\left(x_{0}\right)} u-\inf _{\mathscr{A}_{\varepsilon}^{\delta}\left(x_{0}\right)} u\right) \cdot\left[\left|\partial_{0}^{* *} \mathscr{A}_{\varepsilon_{m}}^{\delta}\left(x_{0}\right)\right|+\left|\partial_{1}^{* *} \mathscr{A}_{\varepsilon_{m}}^{\delta}\left(x_{0}\right)\right|\right] \\
& \cdot\left|\mathscr{A}_{m}\left(k, \eta_{m}\right)\right|^{\left(1 / q_{n_{0}-m-2}\right)-1+(1 / n)-\left(1 / q_{n_{0}}\right)} \\
& +2 \widetilde{C} C_{0}\left(\int_{\overline{A_{0}\left(k, \eta_{0}\right)} \cap \partial_{0}^{* *} \mathscr{A}_{3 \varepsilon / 4}^{\delta}\left(x_{0}\right)}\left|\beta_{1}\right|\left(\eta_{0} u-k\right) d \mathscr{H}_{n-1}\right. \\
& \left.+\int_{\overline{\mathscr{A}_{0}\left(k, \eta_{0}\right)} \cap \partial_{1}^{* *} \mathscr{A}_{3 \varepsilon / 4}^{\delta}\left(x_{0}\right)}|\beta|\left(\eta_{0} u-k\right) d \mathscr{H}_{n-1}\right)\left|\mathscr{A}_{0}\left(k, \eta_{0}\right)\right|^{1 / n-1} \\
& +2^{\tilde{C}_{0}} \cdot \sum_{m=-1}^{n_{0}-1}\left(C_{0}\right)^{m} P_{m} \cdot\left[\left|\int_{\partial \mathscr{A}_{m}\left(k, \eta_{m}\right)} u \frac{\left(D \eta_{m} \cdot \nu_{m}\right)^{-}}{\sqrt{1+\left|D \eta_{m}\right|^{2}}} d \mathscr{H}_{n-1}\right|\right] \\
& \text { - }\left|\mathscr{A}_{m}\left(k, \eta_{m}\right)\right|^{\left(1 / q_{n_{0}-m-2}\right)-1+(1 / n)-\left(1 / q_{n_{0}}\right)} \text {, }
\end{aligned}
$$

analogously to [9, equation (1.22)]. 
4.3. In view of the choice of $\mathscr{A}_{\varepsilon_{m}}^{\delta}\left(x_{0}\right)$ and $\eta_{m}$, we have

$$
\begin{gathered}
\left|\mathscr{A}_{m}\left(k, \eta_{m}\right)\right| \leq \varepsilon^{n-(n-1) \delta}, \quad m=1,2, \ldots, n_{0}, \\
\left|\partial_{i}^{* *} \mathscr{A}_{\varepsilon_{m}}^{\delta}\left(x_{0}\right)\right| \leq \varepsilon^{(n-1)(1-\delta)}, \quad m=1,2, \ldots, n_{0}, \quad i=1,2, \\
\left|\partial_{3}^{* *} \mathscr{A}_{\varepsilon_{m}}^{\delta}\left(x_{0}\right)\right| \leq 2 c_{n} \varepsilon^{(n-1)-(n-2) \delta}, \quad m=1,2, \ldots, n_{0}, \\
P_{m} \leq 2^{(2 / 3)(m+1) m} \cdot \varepsilon^{-(m+1)(1-\delta)} .
\end{gathered}
$$

Moreover, it is easy to see that $n_{0}$ can be chosen to be $n-1$ and $q_{m}, 0 \leq m \leq n_{0}-1$, can be chosen to be arbitrarily close to the number $n /(n-(m+1))$ and $q_{n_{0}}$ can be chosen arbitrarily large; that is, for each positive number $\tilde{\mathcal{\varepsilon}}$, we can choose $q_{m}, 0 \leq m \leq n_{0}-1$, such that

$$
\frac{1}{q_{m}}=\frac{n-(m+1)}{n}-\widetilde{\varepsilon}, \quad \frac{1}{q_{n_{0}}}=\widetilde{\varepsilon} .
$$

With such a choice of $n_{0}$ and $q_{m}, 0 \leq m \leq n_{0}$, we have

$$
\begin{gathered}
P_{n_{0}} \cdot\left|\mathscr{A}_{\varepsilon}^{\delta}\left(x_{0}\right)\right|^{1-\left(1 / q_{n_{0}}\right)+(1 / n)} \leq 2^{\widetilde{C}_{n}} \cdot \varepsilon^{(n+1) \delta / n-\tilde{\varepsilon}}, \\
\sum_{m=-1}^{n_{0}-1}\left(C_{0}\right)^{m} P_{m} \cdot\left(\left|\partial_{0}^{* *} \mathscr{A}_{\varepsilon_{m}}^{\delta}\left(x_{0}\right)\right|+\left|\partial_{1}^{* *} \mathscr{A}_{\varepsilon_{m}}^{\delta}\left(x_{0}\right)\right|\right) \cdot\left|\mathscr{A}_{\varepsilon}^{\delta}\left(x_{0}\right)\right|^{\left(1 / q_{n_{0}-m-2}\right)-1+(1 / n)-\left(1 / q_{n_{0}}\right)} \\
\leq 2^{\widetilde{C}_{n}} \cdot n \cdot\left(C_{0}\right)^{n_{0}} \cdot\left(\varepsilon^{\delta / n-\widetilde{\varepsilon}}+\varepsilon^{(n+1) \delta / n-\tilde{\varepsilon}}\right), \\
\widetilde{C} \cdot\left|\mathscr{A}_{\varepsilon}^{\delta}\left(x_{0}\right)\right|^{(1 / n)-\left(1 / q_{n_{0}}\right)} \leq 2^{\widetilde{C}_{n}} \cdot(n+1) \cdot \varepsilon^{1+(\delta / n)-\widetilde{\varepsilon}},
\end{gathered}
$$

where $\widetilde{C}_{n}=(3 / 2) n(n-1)+2$.

Inserting these into (4.32), we obtain

$$
\begin{aligned}
& \left(\sup _{\mathcal{A}_{\varepsilon / 2}^{\delta}\left(x_{0}\right)} u-\inf _{\mathcal{A}_{\varepsilon / 2}^{\delta}\left(x_{0}\right)} u\right) \leq 2^{\widetilde{C}_{n_{0}}} \cdot C_{0} \cdot \varepsilon^{1-((n-1) \delta / n)-\widetilde{\varepsilon}}+2^{C_{n}} \cdot(n-1) \cdot\left(C_{0}\right)^{n-1} \cdot \varepsilon^{1+(\delta / n)-\widetilde{\varepsilon}} \\
& +2^{C_{n}} \cdot\left(C_{0}\right)^{n-1} \cdot\left(\sup _{\mathscr{A}_{\varepsilon}^{\delta}\left(x_{0}\right)} u-\inf _{\mathscr{A}_{\varepsilon}^{\delta}\left(x_{0}\right)} u\right) \cdot\left(n \varepsilon^{\delta / n-\widetilde{\varepsilon}}+\varepsilon^{1 / n-\widetilde{\varepsilon}}+\varepsilon^{(n+1) \delta / n-\widetilde{\epsilon}}\right) \\
& +2^{C_{n}} \cdot\left(C_{0}\right)^{n-1} \cdot\left(\sup _{\mathscr{A}_{\varepsilon}^{\delta}\left(x_{0}\right)} u-\inf _{\mathscr{A}_{\varepsilon}^{\delta}\left(x_{0}\right)} u\right) \\
& \cdot\left[(\varepsilon)^{(1-n)+(n-1)^{2} \delta / n}\left|\partial \mathscr{A}_{0}\left(k, \eta_{0}\right)\right|+\varepsilon^{(n-1) \delta / n-\tilde{\varepsilon}}\left|\partial \mathscr{A}_{m}\left(k, \eta_{m}\right)\right|\right]
\end{aligned}
$$

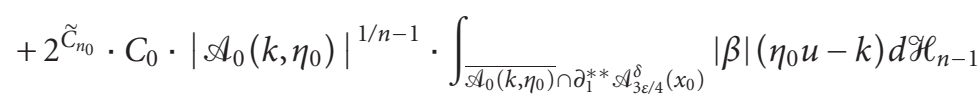

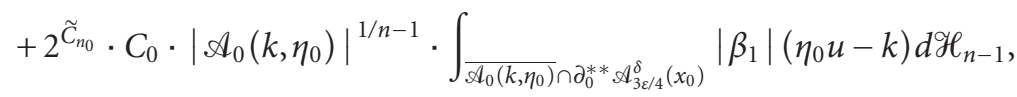

where $C_{n}=5+\left(3 n^{2} / 2\right)+\sum_{m=0}^{n-2}(n /(n-(m+1)))$. 
In view of the rotational symmetry of $\Omega$ and $u$, we choose $\eta_{m}$ to be of the same rotational symmetry so that the level sets of $\eta_{m} u$ are of the same rotational symmetry. Hence, $\partial \mathscr{A}_{m}\left(k, \eta_{m}\right)$ consists of $\partial^{*} \mathscr{A}_{\varepsilon_{m-1}}^{\delta}$, and another region which is parallel to $\partial^{*} \mathscr{A}_{\varepsilon_{m-1}}^{\delta}$, together with some other portion of $\partial \mathscr{A}_{\varepsilon_{i}}\left(x_{0}\right) \subset \partial^{*} \mathscr{A}_{\varepsilon_{m-1}}^{\delta}$. Hence,

$$
\left|\partial \mathscr{A}_{m}\left(k, \eta_{m}\right)\right| \leq 2 \varepsilon^{n-1}+2 \varepsilon^{(n-1)(1-\delta)}+2 c_{n} \varepsilon^{(n-1)-(n-2) \delta} .
$$

4.4. To estimate the last terms in (4.36), we observe that

$$
\begin{aligned}
& \int_{\overline{\mathcal{A}_{0}\left(k, \eta_{0}\right)} \cap \partial_{0}^{* *} \mathscr{A}_{\mathcal{B} \varepsilon / 4}^{\delta}\left(x_{0}\right)}\left|\beta_{1}\right|\left(\eta_{0} u-k\right) d \mathcal{H}_{n-1} \\
& =\int_{\overline{\mathscr{A}_{0}\left(k, \eta_{0}\right)} \cap \partial_{3}^{* *} \mathscr{A}_{3 \varepsilon / 4}^{\delta}\left(x_{0}\right)}\left|\beta_{1}\right|\left(\eta_{0} u-k\right) d \mathscr{H}_{n-1}+\int_{\overline{\mathcal{A}_{0}\left(k, \eta_{0}\right)} \cap \partial_{2}^{* *} \mathscr{A}_{3 \varepsilon / 4}^{\delta}\left(x_{0}\right)}\left|\beta_{1}\right|\left(\eta_{0} u-k\right) d \mathscr{H}_{n-1}, \\
& \int_{\overline{\mathcal{A}_{0}\left(k, \eta_{0}\right)} \cap \partial_{2}^{* *} \mathscr{A}_{3 \varepsilon / 4}^{\delta}\left(x_{0}\right)}\left|\beta_{1}\right|\left(\eta_{0} u-k\right) d \mathscr{H}_{n-1} \\
& \leq\left(\sup _{\mathscr{A}_{\varepsilon}^{\delta}\left(x_{0}\right)} u-\inf _{\mathscr{A}_{\varepsilon}^{\delta}\left(x_{0}\right)} u\right)\left|\partial_{3}^{* *} \mathscr{A}_{3 \varepsilon / 4}^{\delta}\left(x_{0}\right)\right| \leq 2\left(\sup _{\mathscr{A}_{\varepsilon}^{\delta}\left(x_{0}\right)} u-\inf _{\mathscr{A}_{\varepsilon}^{\delta}\left(x_{0}\right)} u\right) c_{n} \varepsilon^{(n-1)-(n-2) \delta} .
\end{aligned}
$$

Hence,

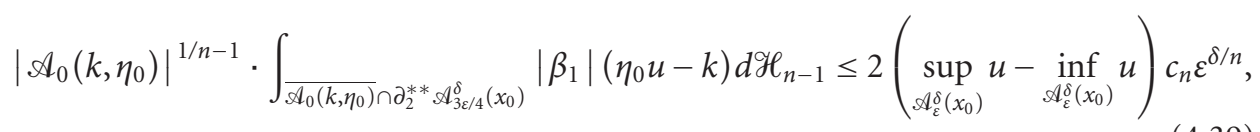

and setting $\eta=1$ in (1.7) with $\Omega$ replaced by $\mathscr{A}_{3 \varepsilon / 4}^{\delta}\left(x_{0}\right)$, we obtain

$$
\begin{aligned}
& \int \overline{\mathcal{A}_{0}\left(k, \eta_{0}\right)} \cap \partial_{3}^{* *} \mathscr{A}_{3 \varepsilon / 4}^{\delta}\left(x_{0}\right) \\
& \quad \leq\left(\sup _{\mathscr{A}_{\varepsilon}^{\delta}\left(x_{0}\right)} u-\inf _{\mathscr{A}_{\varepsilon}^{\delta}\left(x_{0}\right)} u\right) \int_{\overline{A_{0}\left(k, \eta_{0}\right)} \cap \partial_{3}^{* *} \mathscr{A}_{3 \varepsilon / 4}^{\delta}\left(x_{0}\right)}\left|\beta_{1}\right| d \mathscr{H}_{n-1},
\end{aligned}
$$


3948 Boundary behavior of capillary surfaces

in which

$$
\begin{aligned}
& \int_{\overline{\mathcal{A}_{0}\left(k, \eta_{0}\right)} \cap \partial_{3}^{* *} \mathscr{A}_{3 \varepsilon / 4}^{\delta}\left(x_{0}\right)}\left|\beta_{1}\right| d \mathscr{H}_{n-1} \\
& \leq \int_{\mathscr{A}_{\xi \varepsilon / 4}^{\delta}\left(x_{0}\right)} H d x+\int_{\overline{\mathcal{A}_{0}\left(k, \eta_{0}\right)} \cap \partial_{2}^{* *} \mathscr{A}_{\xi \varepsilon / 4}^{\delta}\left(x_{0}\right)}\left|\beta_{1}\right| d \mathcal{H}_{n-1}+\int_{\overline{\mathscr{A}_{0}\left(k, \eta_{0}\right)} \cap \partial_{1}^{* *} \mathscr{A}_{\mathcal{B}_{\varepsilon / 4}\left(x_{0}\right)}^{\delta}}|\beta| d \mathscr{H}_{n-1} \\
& \leq \sup _{\mathscr{A}_{3 \varepsilon / 4}^{\delta}\left(x_{0}\right)}|H|\left|\mathscr{A}_{3 \varepsilon / 4}^{\delta}\left(x_{0}\right)\right|+\int_{\overline{\mathscr{A}_{0}\left(k, \eta_{0}\right)} \cap \partial_{2}^{* *} \mathscr{A}_{\xi \varepsilon / 4}^{\delta}\left(x_{0}\right)}\left|\beta_{1}\right| d \mathscr{H}_{n-1} \\
& +\int_{\overline{\mathcal{A}_{0}\left(k, \eta_{0}\right)} \cap \partial_{1}^{* *} \mathscr{A}_{\mathcal{B}^{\prime} / 4}^{\delta}\left(x_{0}\right)}|\beta| d \mathcal{H}_{n-1} \\
& \leq \sup _{\mathscr{A}_{3 \varepsilon / 4}^{\delta}\left(x_{0}\right)}|H| \varepsilon^{n-(n-1) \delta}+2 c_{n} \varepsilon^{(n-1)-(n-2) \delta}+\int_{\overline{\mathcal{A}_{0}\left(k, \eta_{0}\right)} \cap \partial_{1}^{* *} \mathscr{A}_{3 \varepsilon / 4}^{\delta}\left(x_{0}\right)}|\beta| d \mathscr{H}_{n-1}, \\
& \int_{\overline{\mathcal{A}_{0}\left(k, \eta_{0}\right)} \cap \partial_{1}^{* *} \mathscr{A}_{3 \varepsilon / 4}^{\delta}\left(x_{0}\right)}|\beta|\left(\eta_{0} u-k\right) d \mathscr{H}_{n-1} \\
& \leq\left(\sup _{\mathscr{A}_{\varepsilon}^{\delta}\left(x_{0}\right)} u-\inf _{\mathscr{A}_{\varepsilon}^{\delta}\left(x_{0}\right)} u\right) \int_{\frac{\mathscr{A}_{0}\left(k, \eta_{0}\right)}{\cap} \partial_{1}^{* *} \mathscr{A}_{\mathcal{H}_{\varepsilon / 4}}^{\delta}\left(x_{0}\right)}|\beta| d \mathscr{H}_{n-1},
\end{aligned}
$$

in which

$$
\int_{\overline{\mathcal{A}_{0}\left(k, \eta_{0}\right)} \cap \partial_{1}^{* *} \mathscr{A}_{3 \varepsilon / 4}^{\delta}\left(x_{0}\right)}|\beta| d \mathscr{H}_{n-1} \leq \sup _{\partial_{1}^{* *} \mathscr{A}_{3 \varepsilon / 4}^{\delta}\left(x_{0}\right)}|\beta|\left|\partial_{1}^{* *} \mathscr{A}_{3 \varepsilon / 4}^{\delta}\left(x_{0}\right)\right| \leq \sup _{\partial_{1}^{* *} \mathscr{A}_{3 \varepsilon / 4}^{\delta}\left(x_{0}\right)}|\beta| \varepsilon^{(n-1)(1-\delta)} .
$$

Hence,

$$
\begin{gathered}
\left|\mathscr{A}_{0}\left(k, \eta_{0}\right)\right|^{1 / n-1} \int_{\overline{\mathcal{A}_{0}\left(k, \eta_{0}\right)} \cap \partial_{1}^{* *} \mathscr{A}_{3 \varepsilon / 4}^{\delta}\left(x_{0}\right)}|\beta|\left(\eta_{0} u-k\right) d \mathscr{H}_{n-1} \\
\quad \leq\left(\sup _{\mathscr{A}_{\varepsilon}^{\delta}\left(x_{0}\right)} u-\inf _{\mathscr{A}_{\varepsilon}^{\delta}\left(x_{0}\right)} u\right) \sup _{\partial_{1}^{* *} \mathscr{A}_{\mathcal{B} / 4}^{\delta}\left(x_{0}\right)}|\beta| \mathcal{E}^{-((n-1) / n) \delta .}
\end{gathered}
$$

We have, for sufficiently small $\varepsilon$,

$$
\sup _{\partial_{1}^{* *} A_{3 \varepsilon / 4}^{\delta}\left(x_{0}\right)}|\beta| \leq L\left(\operatorname{diam} \mathscr{A}_{3 \varepsilon / 4}^{\delta}\left(x_{0}\right)\right) \leq L \varepsilon^{1-\delta}
$$

in case $\beta\left(x_{0}\right)=0$ and $\beta$ is Lipschitz continuous with Lipschitz constant $L$ in a neighborhood of $x_{0}$ on $\partial \Omega$, and then, by (4.43),

$$
\left|\mathscr{A}_{0}\left(k, \eta_{0}\right)\right|^{1 / n-1} \int_{\overline{\mathcal{A}_{0}\left(k, \eta_{0}\right)} \cap \partial_{1}^{* *} \mathscr{A}_{3 \varepsilon / 4}^{\delta}\left(x_{0}\right)}|\beta|\left(\eta_{0} u-k\right) d \mathscr{H}_{n-1} \leq L \varepsilon^{1-\delta-((n-1) / n) \delta} .
$$


Inserting (4.45) into (4.41) and afterwards inserting the resultant inequalities into (4.40), we obtain

$$
\begin{aligned}
& \left|\mathscr{A}_{0}\left(k, \eta_{0}\right)\right|^{1 / n-1} \int_{\overline{\mathcal{A}_{0}\left(k, \eta_{0}\right)} \cap \partial_{2}^{* *} \mathcal{A}_{3 \varepsilon / 4}^{\delta}\left(x_{0}\right)}\left|\beta_{1}\right|\left(\eta_{0} u-k\right) d \mathscr{H}_{n-1} \\
& \quad \leq\left(\sup _{\mathcal{A}_{\varepsilon}^{\delta}\left(x_{0}\right)} u-\inf _{\mathscr{A}_{\varepsilon}^{\delta}\left(x_{0}\right)} u\right)\left[\sup _{\mathscr{A}_{3 \varepsilon / 4}^{\delta}\left(x_{0}\right)}|H| \varepsilon^{1-((n-1) / n) \delta}+2 c_{n} \varepsilon^{\delta / n}+L \varepsilon^{1-\delta-((n-1) / n) \delta}\right] .
\end{aligned}
$$

Inserting (4.38) and (4.46) into (4.37) and afterwards inserting the resultant inequality and (4.37) into (4.36), we obtain

$$
\begin{aligned}
& \left(\sup _{\mathscr{A}_{\varepsilon / 2}^{\delta}\left(x_{0}\right)} u-\inf _{\mathscr{A}_{\varepsilon / 2}^{\delta}\left(x_{0}\right)} u\right) \leq 2^{\widetilde{C}_{n_{0}}} \cdot C_{0} \cdot \varepsilon^{1-((n-1) \delta / n)-\widetilde{\varepsilon}} \\
& +2^{C_{n}} \cdot(n-1) \cdot\left(C_{0}\right)^{n-1} \cdot \varepsilon^{1+(\delta / n)-\tilde{\varepsilon}} \\
& +2^{C_{n}} \cdot\left(C_{0}\right)^{n-1} \cdot\left(\sup _{\mathcal{A}_{\varepsilon}^{\delta}\left(x_{0}\right)} u-\inf _{\mathscr{A}_{\varepsilon}^{\delta}\left(x_{0}\right)} u\right) \cdot\left(n \varepsilon^{\delta / n-\tilde{\varepsilon}}+\varepsilon^{(n+1) \delta / n-\widetilde{\varepsilon}}\right) \\
& +2^{\widetilde{C}_{n_{0}}+2} \cdot C_{0} \cdot c_{n} \cdot\left(\sup _{\mathscr{A}_{\varepsilon}^{\delta}\left(x_{0}\right)} u-\inf _{\mathscr{A}_{\varepsilon}^{\delta}\left(x_{0}\right)} u\right) \cdot \varepsilon^{\delta / n} \\
& +2^{\widetilde{C}_{n_{0}}+2} \cdot C_{0} \cdot \sup _{A_{3 \varepsilon / 4}^{\delta}\left(x_{0}\right)}|H| \cdot\left(\sup _{\Omega} u-\inf _{\Omega} u\right) \cdot \varepsilon^{1-((n-1) / n) \delta} \\
& +2^{\widetilde{C}_{n_{0}}+1} \cdot C_{0} \cdot L \cdot \varepsilon^{1-\delta-((n-1) / n) \delta} .
\end{aligned}
$$

Choose $\delta=1 / 2$. We obtain

$$
\left(\sup _{\mathscr{A}_{\varepsilon / 2}^{\delta}\left(x_{0}\right)} u-\inf _{\mathscr{A}_{\varepsilon / 2}^{\delta}\left(x_{0}\right)} u\right) \leq \widetilde{C}_{n} \varepsilon^{1 / 2 n}
$$

where

$$
\tilde{C}_{n}=2^{C_{n}+2} n \cdot\left(C_{0}\right)^{n-1} \cdot\left(\sup _{\Omega} u-\inf _{\Omega} u\right)\left(1+c_{n}+L+\sup _{\mathbb{A}_{3 \varepsilon / 4}^{\delta}\left(x_{0}\right)}|H|\right) .
$$

This completes the proof of Main Theorem 1.2.

\section{Acknowledgment}

I wish to thank the referee for careful reading and precious suggestions. 


\section{References}

[1] M. Emmer, Esistenza, unicità e regolarità nelle superfici de equilibrio nei capillari, Ann. Univ. Ferrara Sez. VII (N.S.) 18 (1973), 79-94.

[2] R. Finn, Equilibrium Capillary Surfaces, Fundamental Principles of Mathematical Sciences, vol. 284, Springer, New York, 1986.

[3] C. Gerhardt, Existence and regularity of capillary surfaces, Boll. Un. Mat. Ital. (4) 10 (1974), $317-335$.

[4] Existence, regularity, and boundary behavior of generalized surfaces of prescribed mean curvature, Math. Z. 139 (1974), no. 2, 173-198.

[5] - On the capillarity problem with constant volume, Ann. Scuola Norm. Sup. Pisa Cl. Sci. (4) 2 (1975), no. 2, 303-320.

[6] E. Giusti, On the equation of surfaces of prescribed mean curvature. Existence and uniqueness without boundary conditions, Invent. Math. 46 (1978), no. 2, 111-137.

[7] , Generalized solutions for the mean curvature equation, Pacific J. Math. 88 (1980), no. 2, 297-321.

[8] N. Korevaar and L. Simon, Equations of mean curvature type with contact angle boundary conditions, Geometric Analysis and the Calculus of Variations (J. Jost, ed.), International Press, Massachusetts, 1996, pp. 175-201, Dedicated to Stefan Hildebrandt on the occasion of his 60th birthday.

[9] F.-T. Liang, Interior Hölder estimates for non-parametric surfaces of prescribed mean curvature, Proc. Roy. Soc. Edinburgh Sect. A 133 (2003), no. 3, 625-638.

[10] Global boundedness, interior gradient estimates, and boundary regularity for the mean curvature equation with boundary conditions, Int. J. Math. Math. Sci. 2004 (2004), no. 18, 913-948.

[11] _ Boundary regularity for capillary surfaces, Georgian Math. J. 12 (2005), no. 2, 283-307.

[12] V. G. Maz'ya, Sobolev Spaces, Springer Series in Soviet Mathematics, Springer, Berlin, 1985.

[13] M. Miranda, Superficie minime illimitate, Ann. Scuola Norm. Sup. Pisa Cl. Sci. (4) 4 (1977), no. 2, 313-322.

[14] L. Simon, Global estimates of Hölder continuity for a class of divergence-form elliptic equations, Arch. Ration. Mech. Anal. 56 (1974), no. 3, 253-272.

[15] L. Simon and J. Spruck, Existence and regularity of a capillary surface with prescribed contact angle, Arch. Ration. Mech. Anal. 61 (1976), no. 1, 19-34.

[16] G. Stampacchia, Èquations elliptiques du second ordre à coefficients discontinus, Séminaire de Mathématiques Supérieures, No. 16 (Été, 1965), Les Presses de l’Université de Montréal, Montréal, 1966.

Fei-Tsen Liang: Institute of Mathematics, Division of Mathematics and Physical Sciences, Academia Sinica, Nankang, Taipei 11529, Taiwan

E-mail address: liang@math.sinica.edu.tw 


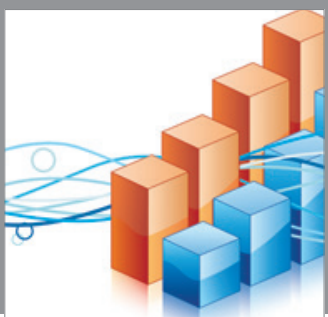

Advances in

Operations Research

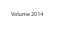

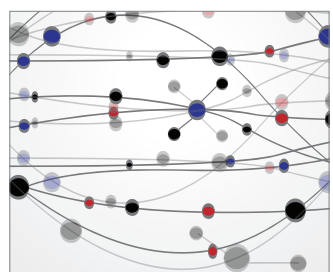

\section{The Scientific} World Journal
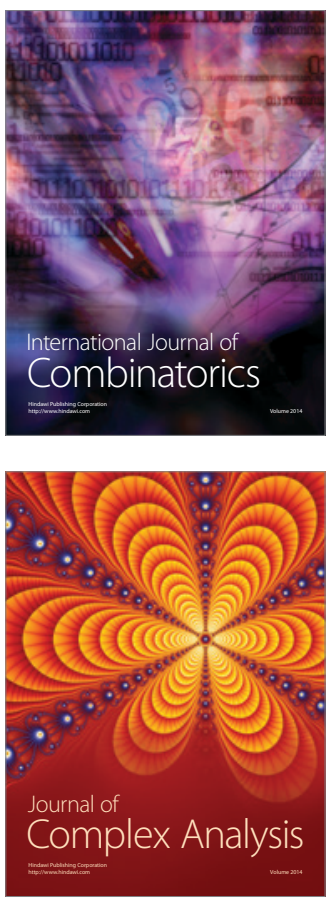

International Journal of

Mathematics and

Mathematical

Sciences
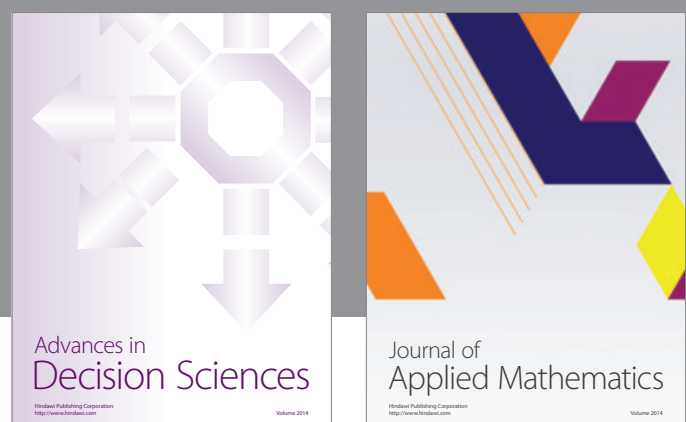

Journal of

Applied Mathematics
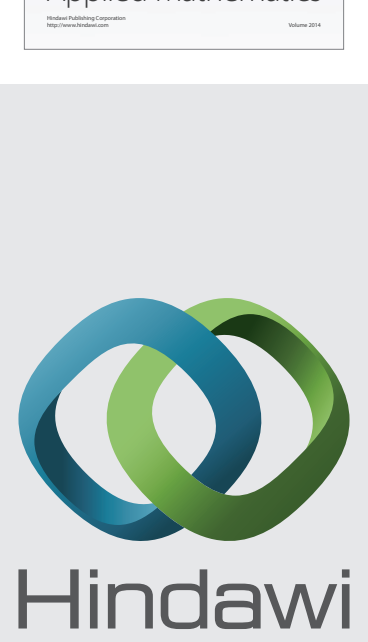

Submit your manuscripts at http://www.hindawi.com
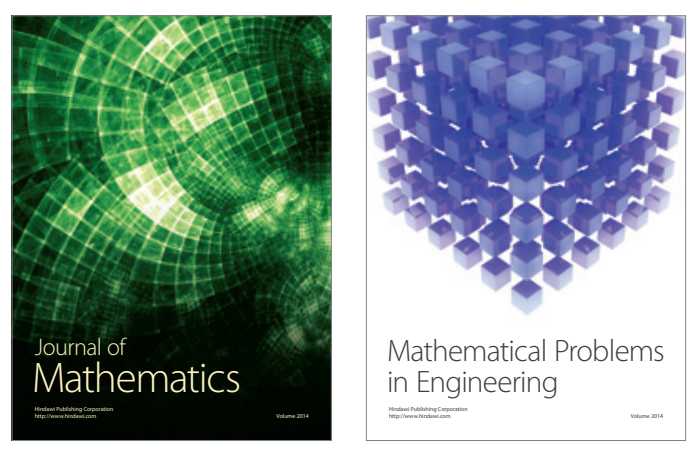

Mathematical Problems in Engineering
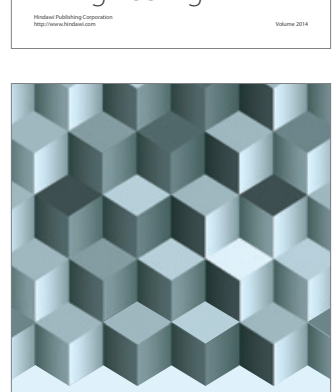

Journal of

Function Spaces
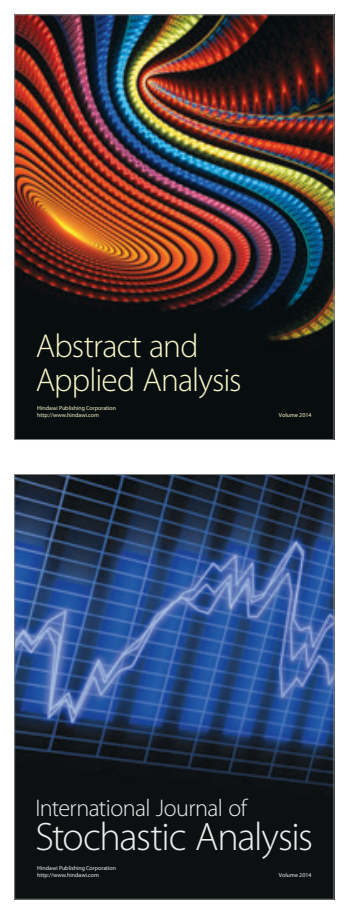

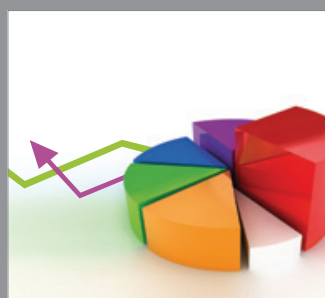

ournal of

Probability and Statistics

Promensencen
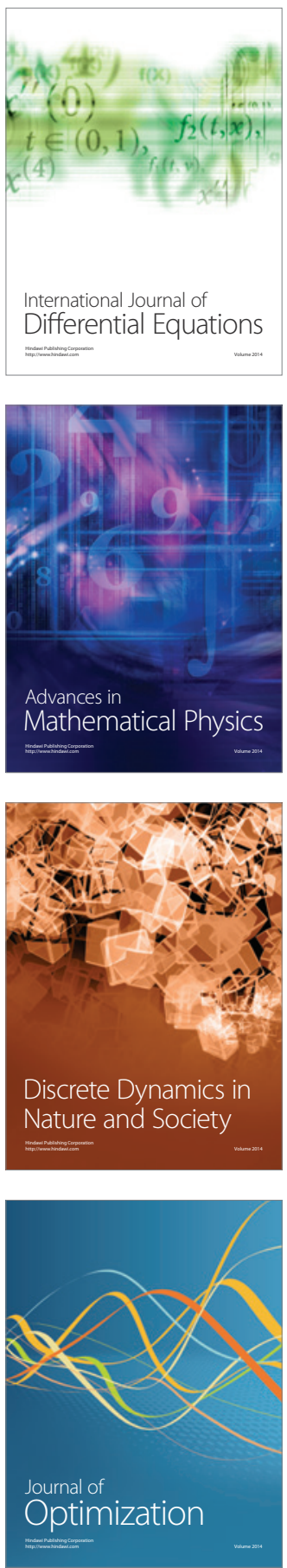